\title{
The energy dependence of the $\pi N$ amplitude and the three-nucleon interaction
}

\author{
T-Y. Saito and I. R. Afnan \\ School of Physical Sciences, The Flinders University of South Australia, \\ Bedford Park, SA 5042, Australia
}

(October 23, 2018)

\begin{abstract}
By calculating the contribution of the $\pi-\pi$ three-body force to the threenucleon binding energy in terms of the $\pi N$ amplitude using perturbation theory, we are able to determine the importance of the energy dependence and the contribution of the different partial waves of the $\pi N$ amplitude to the three-nucleon force. A separable representation of the non-pole $\pi N$ amplitude allows us to write the three-nucleon force in terms of the amplitude for $N N \rightarrow$ $N N^{*}$, propagation of the $N N N^{*}$ system, and the amplitude for $N N^{*} \rightarrow$ $N N$, with $N^{*}$ being the $\pi N$ quasi-particle amplitude in a given state. The division of the $\pi N$ amplitude into a pole and non-pole gives a procedure for the determination of the $\pi N N$ form factor within the model. The total contribution of the three-body force to the binding energy of the triton for the separable approximation to the Paris nucleon-nucleon potential (PEST) is found to be very small mainly as a result of the energy dependence of the $\pi N$ amplitude, the cancellation between the $S$ - and $P$-wave $\pi N$ amplitudes, and the soft $\pi N N$ form factor.
\end{abstract}




\section{INTRODUCTION}

The discrepancy between the results of the exact calculations for the binding energy of the triton using a number of realistic nucleon-nucleon potentials and the experimental value of $8.48 \mathrm{MeV}$, has been an outstanding problem in nuclear physics for a number of years [1]. A commonly accepted solution has been the introduction of a three-nucleon force that will bridge the gap between the calculated binding energy [2 [5], based on a two-body interaction, and the experimental binding energy. The origin of such a three-body force is partly the result of the fact that the nucleons are treated as point particles interacting via a two-body meson exchange potential that is often assumed to be local. The fact that the formal division of the interaction into a two- and three-body part is not unique, given the on-shell two-body data [6], suggests that the contribution of the three-body force is partly determined by the definition of the two-nucleon interaction. Thus under ideal conditions the division of the interaction, in the three-nucleon system, between a two- and three-body force will require a consistent formulation of these two potentials within a meson-nucleon theory.

In the absence of such a formulation one may assume that a meson-nucleon theory should give the correct binding energy for the three-nucleon system, in which case the three-body force is by definition that force which when added to the chosen two-nucleon force will give the three-nucleon binding energy [7 9]. A second approach is to assume that the threenucleon force is the result of meson exchanges that are possible only when the number of nucleons is greater than two. In this second approach one expects the dominant mechanism to be one in which one nucleon emits a meson that scatters off a second nucleon and then gets absorbed on the third nucleon, see Fig. 1. In this case the three-nucleon force is determined by the off-shell meson-nucleon amplitude that goes into the calculation of the diagram in Fig. 11. In the present investigation we will consider the second approach involving a $\pi$ meson exchange. In particular, we will examine the role of the energy dependence of this $\pi N$ amplitude on the contribution of this three-body force to the binding energy of the triton. We will also examine the relative contribution of the different $\pi N$ partial waves to this three-body force.

Over the past ten years three approaches have been developed to determine a threenucleon interaction from $\pi N$ dynamics. (i) The Tucson-Melbourne (TM) [10,11] threenucleon potential is based on the idea that the off-mass shell $\pi N$ amplitude should satisfy current algebra constraints and the soft pion theorems [12]. These constraints allow a covariant parameterization of the off-mass shell amplitude. To be consistent with the meson exchange $N N$ interaction, the $\pi N$ amplitude is expanded in powers of $\frac{q}{m_{N}}$, where $q$ is the pion momentum and $m_{N}$ the nucleon mass. This gives a $\pi N$ amplitude that includes both $S$ and $P$-wave scattering, but where the energy dependence is reduced to $\nu=(s-u) / 4 m_{N}=$ $\left(q^{\prime}+q\right) \cdot\left(p^{\prime}+p\right) / 4 m_{N}=0$, as a result of the expansion in $\frac{q}{m_{N}}$. Here, $s$ and $u$ are the usual Mandelstam variables while $q\left(q^{\prime}\right)$ and $p\left(p^{\prime}\right)$ are the initial (final) four momentum of the pion and nucleon respectively, The $\pi N N$ form factor is constructed to satisfy the Goldberger-Treiman relation [13,14]. Although the original TM potential included only the $\pi-\pi$ three-body force, $\pi-\rho$ and $\rho-\rho$ contributions have recently been included [15. (ii) A similar approach is to assume that the $\pi N$ dynamics is determined by an effective chiral Lagrangian 16 20] which when used to calculate the $\pi N$ amplitude at the tree level, 
will give an effective three-nucleon force given by the diagram in Fig. 11. The evaluation of the $\pi N$ amplitude at the tree level gives rise to an energy independent $\pi N$ amplitude, and therefore a three-body force. Both approaches (i) and (ii) give similar results, and emphasize the chiral symmetry of the $\pi N$ amplitude. (iii) The $N-\Delta$ coupled channel approach 221 25] takes as its starting point the fact that the $\pi N$ amplitude is dominated at medium energies by the $\Delta(1230)$ or the $P_{33}$ partial wave. This dominance of the $\Delta$ suggests that we could extend our Hilbert space to include not only the nucleon, but also the $\Delta$ as an excited state of the nucleon. The approach of treating the $N$ and $\Delta$ on equal footing effectively includes, in a consistent manner, that part of the original three-body force corresponding to $\pi N$ scattering in the $P_{33}$ channel, or at least the resonance part of it [26]. The advantage of this approach is that now we can construct the two-body and the three-body forces with some consistency, to the extent that the $N N-N \Delta$ transition potential used in the two-body interaction can also be used to generate the three-body potential. The inclusion of coupling between the $N N, N \Delta$ and $\Delta \Delta$ channels allows a consistent treatment of the $B B$ and $B B B$ system, where $B=N, \Delta$ [25]. In this approach, since the $\pi N$ amplitude is basically approximated by $\pi N \rightarrow \Delta \rightarrow \pi N$, the energy dependence of the total $\pi N$ amplitude is completely determined by the energy dependence of the $P_{33}$ channel which has the $\Delta$ resonance. Although the $N N$ and transition potentials can be local and energy independent, the effective three-body force in this model is energy dependent and this energy dependence is determined by the $\Delta$ resonance, i.e. the $P_{33}$ amplitude. More recently there have been extensions of the $N-\Delta$ coupled channel approach that have included the $S$-wave component of the TM potential [26]. Also Peña et al. [27] have examined the importance of the coupling of the $\Delta$ to the $\pi N$ channel. This latter calculation gives a $\pi N$ amplitude in the $P_{33}$ channel that fits the phase shifts and has an energy dependent mass and width for the $\Delta$. The inclusion of this energy dependence in the $\Delta$ mass and width does not effect the final result appreciably.

The questions which arise from the above approaches to the $\pi N$ dynamics that go into the derivation of the three-nucleon force and its contribution to the binding energy of the three-nucleon system are: (i) What are the contributions of the different $\pi N$ partial waves to the three-body force? (ii) If there is any cancellation between the contribution of the different partial waves, should the $P_{33}$ partial wave be treated via the $N-\Delta$ coupled channel while the rest of the $\pi N$ amplitude gives rise to a three-body force as depicted in Fig. 田? (iii) Would a cancellation between the different $\pi N$ channels be sensitive to the energy dependence of the amplitudes? The main aim of this investigation is to examine these questions.

To motivate our interest in the importance of the energy dependence of the $\pi N$ amplitude, let us examine the role of the $N N$ amplitude in calculating the binding energy of the three-nucleon system within the framework of the Faddeev equations. Here we observe that we require the fully off-energy-shell $N N$ amplitude in a given partial wave $\alpha$, $t_{\alpha}^{N N}\left(k, k^{\prime} ; E_{N N}\right)$, for all energies in the range $-\infty<E_{N N}<-E_{T}$, where $E_{T}$ is the threenucleon binding energy, see Fig. 2. The fact that we need this amplitude over the full specified energy domain is a result of the fact that in the three-nucleon system, the total energy is fixed at $E=-E_{T}$, and the spectator particle can have any energy from zero to $\infty$. To consider the contribution of Fig. [1 and in particular the energy dependence of the $\pi N$ amplitude, we consider the $N N N-\pi N N N$ equations [28,29] which are an extension of the $N N-\pi N N$ equations to the $A=3$ sector. Within the framework of the above $\pi N N$ 
dynamics, to calculate the contribution of the three-body force as defined in Fig. 1 to the three-nucleon binding, we must determine to know the fully off-energy-shell $\pi N$ amplitudes [30], $t_{\alpha}^{\pi N}\left(k, k^{\prime} ; E_{\pi N}\right)$, for all energies in the range $-\infty<E_{\pi N}<\left(m_{N}-E_{T}\right)$, where in this case we have included the rest mass of the nucleon and pion in the $\pi N$ energy $E_{\pi N}$. In other words we have to calculate the fully off-energy-shell $\pi N$ amplitude for all energies from $-\infty$ to $E_{T}$ below the nucleon pole ( see Fig. (3). Thus to calculate a three-body force which is defined in terms of the $\pi N$ amplitude, we need to know this amplitude off-shell for energies far below $\left(\left(m_{\pi}+E_{T}\right) \approx 145 \mathrm{MeV}\right)$, the $\pi N$ threshold. Here the need for the $\pi N$ amplitude over this energy domain is a result of the fact that the total energy is still $-E_{T}$, but now we have two spectator nucleons with kinetic energy between zero and $\infty$. At these energies it is not clear that the $\Delta(1230)$ is dominant or that the $P$-wave amplitudes are more important than the $S$-wave amplitudes. Clearly, the threshold behavior of the amplitude is essential, but we need to know the $\pi N$ partial wave amplitudes over a wide range of energies above threshold, if we are to extrapolate these amplitudes to the energies required in calculating the three-body force. This suggests that we have to fit the energy dependence of the experimental $\pi N$ phase shifts in order to improve the accuracy of the extrapolation in the energy.

In Sec. II we will derive the three-body force using the coupled channel method and we will discuss approximation which we take to avoid the dressing problem [31. In Sec. [II we will discuss a parameterization for the $\pi N$ amplitude, and in particular will discuss the division of this amplitude into a pole and non-pole component. It is only the non-pole part of the $\pi N$ amplitude that goes into the $\pi N$ scattering that generates the three-body force. This division is essential if we are to avoid double counting. We will choose the $\pi N N$ form factor as the residue of the pole term in the $\pi N$ amplitude to keep the consistency with the $\pi N$ formulation. We will then proceed in Sec. $\mathbb{\nabla}$ to a discussion of the $\pi-\pi$ three-body force given in Fig. 1 in terms of our parameterization of the $\pi N$ amplitude. As a result of using a separable representation for the $\pi N$ amplitude, we find that the three-body force is reduced to the product of the amplitude for $N N \rightarrow N N_{\alpha}^{*}$, followed by the propagation of the $N N N_{\alpha}^{*}$ system, and the final transition amplitude for $N N_{\alpha}^{*} \rightarrow N N$, where $\alpha$ runs over all $\pi N$ partial waves. This $\pi-\pi$ three-body force is employed in Sec. $\nabla$ to calculate, in the Born approximation, the contribution of this force to the binding energy of the three-nucleon system. The three-nucleon wave function will be calculated by using Paris (PEST) potential [32]. Since the Paris potential does not have any energy dependence, our approach for the three-nucleon interaction is inconsistent with the two-nucleon interaction. To that extent, the overall magnitude of the resultant three-body force contribution may not have great significance. However, we would like to examine the relative contribution of the different $\pi N$ partial waves, and see how this contribution is sensitive to the energy dependence of the $\pi N$ amplitude in the subthreshold region. In particular, we would like to demonstrate how the energy dependence of both the non-pole amplitude and the $\pi N N$ form factor play a role in the importance of this three-nucleon force. Here we will find that the energy dependence of the non-pole amplitude has a significant effect on the contribution of the three-body force. Furthermore, we will demonstrate that there is a cancellation between the contribution of the different $\pi N$ partial waves. This cancellation turns out to be very sensitive to the approximations used. Finally, in Sec. VI we will conclude our discussion by considering some open questions that can influence our final results. 


\section{COUPLED CHANNEL FORMULATION OF THREE-BODY FORCE}

In this section, we will establish the approximation involved in writing Fig. 1 as the lowest order contribution to the binding energy of the three-nucleon system from a $\pi-\pi$ three-body force. Our starting point is the Hamiltonian of Mizutani and Koltun [33]

$$
\mathcal{H}=\mathcal{K}+\mathcal{V}_{N N}+\mathcal{V}_{\pi N}+\mathcal{A}+\mathcal{A}^{\dagger}
$$

where $\mathcal{K}$ is the kinetic energy of the nucleons and pions, while $\mathcal{V}_{N N}$ and $\mathcal{V}_{\pi N}$ are the $N N$ interaction in the absence of one pion exchange, and the $\pi N$ interaction in the absence of the s-channel nucleon pole, respectively. In Eq. (2.1), $\mathcal{A}$ is the pion absorption vertex while $\mathcal{A}^{\dagger}$ is the corresponding production vertex. The Schrödinger equation for this Hamiltonian is

$$
\mathcal{H}|\Psi\rangle=E|\Psi\rangle
$$

Clearly, the operators $\mathcal{A}$ and $\mathcal{A}^{\dagger}$ change the number of pions. To that extent, the present Hamiltonian has some of the features of a field theory in that the number of pions is not fixed while the number of nucleons is fixed. As a first approximation, we restrict the Hilbert space to $(n N)$ and $\pi(n N)$ systems only, where $n$ is the number of nucleons, which is conserved. We define the Feshbach [34] projection operators onto $(n N)$ and $\pi(n N)$ spaces as $P$ and $Q$, respectively. By using these projection operators and by assuming that this truncated space is complete, i.e. $P+Q=1$, the wave functions of the $(n N)$ and $\pi(n N)$ components ( $P \Psi$ and $Q \Psi$, respectively) are solutions of the equations

$$
\left(E-H_{P P}-H_{P Q} \frac{1}{E-H_{Q Q}} H_{Q P}\right)|P \Psi\rangle=0,
$$

and

$$
\left(E-H_{Q Q}-H_{Q P} \frac{1}{E-H_{P P}} H_{P Q}\right)|Q \Psi\rangle=0 .
$$

In writing Eqs. (2.3) and (2.4) we have made use of the definitions,

$$
\begin{aligned}
H_{P P} & \equiv P \mathcal{H} P, \\
H_{Q P} & \equiv Q \mathcal{H} P, \quad \text { and } \quad H_{P Q} \equiv P \mathcal{H} Q, \\
H_{Q Q} & \equiv Q \mathcal{H} Q .
\end{aligned}
$$

The Green's function for the $\pi(n N)$ system, $\left(E-H_{Q Q}\right)^{-1}$, which including the $\pi N$ and $N N$ interactions, can be written as

$$
\frac{1}{E-H_{Q Q}}=G_{\pi(n N)}^{(0)}(E)+G_{\pi(n N)}^{(0)}(E) T(E) G_{\pi(n N)}^{(0)}(E)
$$

where $G_{\pi(n N)}^{(0)}(E)$ is the free $\pi(n N)$ propagator. The corresponding T-matrix, $T(E)$, can be written as

$$
T(E)=\sum_{a} t_{a}(E)+\sum_{a b} t_{a}(E) G_{\pi(n N)}^{(0)}(E) U_{a b}(E) G_{\pi(n N)}^{(0)}(E) t_{b}(E)
$$


where $U_{a b}(E)$ is the AGS amplitude [35] for the $(n+1)$ particle system and satisfies a set of coupled equations. Here, $t_{a}(E)$ is the $\pi N$ or $N N$ amplitudes resulting from the potential $\mathcal{V}_{N N}$ or $\mathcal{V}_{\pi N}$, respectively.

We will first consider the simplest case of $n=1$ [36]. Here, on the one hand, Eq.(2.3) can be regarded as the equation for the dressed nucleon with the self-energy, $\Sigma(E)$, defined to be

$$
\Sigma(E)=H_{P Q} \frac{1}{E-H_{Q Q}} H_{Q P} .
$$

Therefore the dressed nucleon propagator, $G_{N}(E)$, can be written as

$$
G_{N}(E)=\left(E-H_{P P}-H_{P Q} \frac{1}{E-H_{Q Q}} H_{Q P}\right)^{-1}
$$

with $H_{P P}$, the bare mass of the nucleon. On the other hand, Eq. (2.4) describes $\pi N$ scattering. Now since $H_{Q Q}=K_{Q Q}+\mathcal{V}_{\pi N}$, with $K_{Q Q}$ the kinetic energy of the $\pi N$ system, the $\pi N$ interaction in Eq. (2.4) is the sum of two contributions. The first is the $\pi N$ interaction in the original Hamiltonian, $\mathcal{V}_{\pi N}$, while the second term, $H_{Q P}\left(E-H_{P P}\right)^{-1} H_{P Q}$, results from the coupling of the $\pi N$ channel to the $N$ channel. The $\pi N$ T-matrix, $T_{\pi N}(E)$, in this case is the sum of two terms, and is given by

$$
T_{\pi N}(E)=F_{Q P}(E) G_{N}(E) F_{P Q}(E)+t_{B}(E)
$$

where

$$
F_{P Q}(E)=H_{P Q}\left[1+\frac{1}{E-K_{Q Q}} t_{B}(E)\right],
$$

and $t_{B}(E)$ is the solution to the two-body equation

$$
t_{B}(E)=V_{Q Q}+V_{Q Q} \frac{1}{E-K_{Q Q}} t_{B}(E)
$$

with $V_{Q Q}=\mathcal{V}_{\pi N}$. Here we note that the first term on the right hand side of Eq. (2.10) corresponds to the process $\pi N \rightarrow N \rightarrow \pi N$, and as a result the pion absorption (emission) vertex is given by $F_{P Q}(E)\left(F_{Q P}(E)\right)$. To establish that this term has the nucleon pole contribution to the $\pi N$ amplitude, we note that the Green's function, $G_{N}(E)$, given in Eq. (2.9), has a pole at the nucleon mass, and therefore can be written as

$$
G_{N}(E)=\frac{1}{E-H_{P P}-\Sigma(E)}=\frac{Z_{2}(E)}{E-m_{N}},
$$

where $Z_{2}(E)$ is the wave function renormalization. Making use of this result in Eq. (2.10) allows us to write the $\pi N$ T-matrix as

$$
T_{\pi N}(E)=f_{\pi N N}^{R \dagger}(E) \frac{1}{E-m_{N}} f_{\pi N N}^{R}(E)+t_{B}(E)
$$

where the renormalized $\pi N N$ form factor, which is energy dependent, is given by 


$$
f_{\pi N N}^{R}(E)=Z_{2}^{\frac{1}{2}}(E) F_{P Q}(E) .
$$

In this formulation the normalized physical nucleon wave function, which is a solution to Eq. (2.3) is given by

$$
\Psi_{N}=Z_{2}^{\frac{1}{2}}\left(m_{N}\right) P \Psi
$$

We consider next the case of $n>1$. Since we have restricted the Hilbert space to include $n$ nucleons, and up to one pion only, this truncation effects the dressing of the nucleons for the case $n>1$. From Fig. 1 , we observe that the nucleons can only be dressed separately after absorbing an initial pion and before emitting the final pion, because of the limitation imposed on the Hilbert space. We can neither include the nucleon dressing before the pion absorption nor after the final pion emission. This incomplete dressing makes the renormalized $\pi N N$ form factor, $f_{\pi N N}^{R}(E)$, smaller [31]. To overcome this problem we need to guarantee that all the nucleons are fully dressed at the same time, and this dressing is on both sides of the $\pi N N$-vertex [37], see Fig. 5. We will avoid this problem in the present investigation in the following manner. In the $N N-\pi N N$ equations the nucleon dressing was introduced to satisfy the unitarity of the $N N$ amplitude above the pion production threshold. In the present investigation however, we are considering a bound state problem which is below the threshold for pion production. We therefore expect that the nucleon dressing will not be essential, and does not have to be included explicitly to satisfy unitarity. We therefore assume that each nucleon line has been renormalized to give the physical nucleon mass and correct $\pi N N$ coupling constant, i.e.; (i) The nucleon propagator is given by $\left(E-m_{N}\right)^{-1}$. (ii) The $\pi N N$ vertex is given by the renormalized vertex function, $f_{\pi N N}^{R}(E)$.

Using the above assumptions, the interaction term resulting from the coupling of the $N N N$ and $\pi N N N$ Hilbert space in Eq. (2.3) can be rewritten as

$$
H_{P Q} \frac{1}{E-H_{Q Q}} H_{Q P}=\mathcal{V}_{O P E}+\mathcal{V}_{d i s p}+\mathcal{V}_{3 B}+\cdots
$$

where the series is generated by iterating the equation for $U_{a b}$ in Eq. (2.7), and making use of the resultant $\pi N N N$ T-matrix in Eq. (2.6). In particular, if we go to third order in the $\pi N$ amplitude $t_{a}$, the $\pi N N$ form factors in $\mathcal{V}_{3 B}$ get dressed as detailed in Eqs. (2.14) and (2.15). Each of the terms in Eq. (2.17) is shown schematically in Fig. 6. We note that $\mathcal{V}_{O P E}$ and $\mathcal{V}_{\text {disp }}$ are two-body operators. Since we will be calculating perturbatively the contribution of the three-body force to the binding energy of ${ }^{3} \mathrm{H}$, the three nucleon wave function used results from the solution of the Faddeev equations for a given nucleon-nucleon interaction. Assuming that the nucleon-nucleon interaction includes $\mathcal{V}_{O P E}$ and $\mathcal{V}_{\text {disp }}$, we omit these terms from Eq. (2.17). This leaves us with the three-nucleon force $\mathcal{V}_{3 B}$, illustrated in Figs. 6(c) and 1, which gives the lowest order correction to the binding energy of the three-nucleon system. The detail of the numerical framework of the $\pi N$ scattering will be presented in next section.

\section{THE $\pi N$ AMPLITUDE}

To include the full energy dependence of the $\pi N$ amplitude into the determination of the $\pi-\pi$ three-nucleon interaction and its contribution to the binding energy of the triton, 
we have to: (i) Remove the nucleon pole contribution from the $\pi N$ amplitude to avoid double counting in the three nucleon force calculation. (ii) Define a $\pi N N$ form factor for the emission and absorption vertices in Fig. 1 that is consistent with the $\pi N$ amplitude used and the scattering data. (iii) Treat the nucleon in the $\pi N$ system using non-relativistic kinematics to maintain consistency between the $N N N$ and $\pi N$ systems since the nucleons in the threenucleon system are treated non-relativistically. From the previous section we observe the first two conditions can be satisfied if we choose a formulation of the $\pi N$ scattering problem that is motivated by a Hamiltonian that includes a $\pi N N$ vertex and a $\pi N$ interaction, e.g. the Cloudy Bag Model [38]. Thus a choice for the $\pi N$ potential, motivated by the lowest order contribution to the amplitude and based on a Lagrangian of the form suggested by the Cloudy Bag Model with volume coupling [39], consists of an $s$-channel nucleon pole diagram, Fig. 7, and the cross diagram and contact term, Fig. 8.

For the present investigation, to simplify the parameterization of the $\pi N$ amplitude, we replace the cross diagram and contact term in Fig. 8 by a one term separable potential in each partial wave. This allows us to write the $\pi N$ potential in a given partial wave $\alpha$ as

$$
v_{\alpha}\left(k, k^{\prime} ; E\right)=f_{0}(k) \frac{1}{E-m_{0}} f_{0}\left(k^{\prime}\right)+g_{\alpha}(k) \lambda_{\alpha} g_{\alpha}\left(k^{\prime}\right),
$$

where the first term corresponds to the nucleon pole diagram with a bare nucleon mass of $m_{0}$, and a bare form factor $f_{0}(k)$. Since we are using non-relativistic kinematics for the nucleon, this $s$-channel pole diagram contributes to the $P_{11}$ partial wave only. As a result, the potential in all partial waves other than the $P_{11}$ channel is given by the second term on the right hand side of Eq. (3.1). This separable $\pi N$ potential gives an amplitude of the form

$$
t_{\alpha}\left(k, k^{\prime} ; E\right)=g_{\alpha}(k) \tau_{\alpha}(E) g_{\alpha}\left(k^{\prime}\right) \quad \text { for } \quad \alpha \neq P_{11}
$$

where

$$
\tau_{\alpha}(E)=\left[\lambda_{\alpha}^{-1}-\left\langle g_{\alpha}\left|G_{\pi N}(E)\right| g_{\alpha}\right\rangle\right]^{-1}
$$

with

$$
\left\langle g_{\alpha}\left|G_{\pi N}(E)\right| g_{\alpha}\right\rangle=\int_{0}^{\infty} d k k^{2} \frac{\left[g_{\alpha}(k)\right]^{2}}{E-\omega_{k}-\frac{k^{2}}{2 m_{N}}-m_{N}}
$$

This amplitude is a solution of the Lippmann-Schwinger equation with the pion energy treated relativistic since $\omega_{k}=\sqrt{k^{2}+m_{\pi}^{2}}$. The strength of the potential $\lambda_{\alpha}$, and the form factor $g_{\alpha}(k)$ are adjusted to fit the experimental $\pi N$ phase shifts in all $S$ - and $P$-waves other than the $P_{11}$ channel. For the present investigation we will use the parameterization used by Thomas [40] for $\pi d$ scattering. The form factor used for $S_{11}$ and $S_{31} \pi N$ partial waves is:

$$
g_{\alpha}(k)=\frac{S_{1}}{k^{2}+\alpha_{1}^{2}}+\frac{S_{2}}{k^{2}+\alpha_{2}^{2}},
$$

and

$$
g_{\alpha}(k)=k\left[\frac{S_{1}}{k^{2}+\alpha_{1}^{2}}+\frac{S_{2} k^{2}}{\left(k^{2}+\alpha_{2}^{2}\right)^{2}}\right]
$$


for $P_{13}, P_{31}$ and $P_{33}$. This parameterization has also been used extensively in the $N N-\pi N N$ calculation for $\pi d$ scattering and $\pi d \rightarrow p p$ reactions [41,42].

The $P_{11}$ channel plays the important role in this analysis of the three-nucleon interaction as it has the nucleon pole contribution that needs to be removed to avoid double counting. It also has the information about the $\pi N N$ form factor which is defined as the residue of the off-shell $\pi N$ amplitude at the nucleon pole. In this way we can subtract the nucleon pole contribution to the $\pi N$ amplitude, and extract a $\pi N N$ form factor while maintaining a fit to the $\pi N$ scattering data in this channel. Since the potential in this channel is the sum of two contributions, see Eq. (3.1), we can write the corresponding amplitude as a solution of the Lippmann-Schwinger equation using a two-potential theory to be 43.

$$
t_{\alpha}\left(k, k^{\prime} ; E\right)=f(k ; E) \frac{1}{E-m_{0}-\Sigma(E)} f\left(k^{\prime} ; E\right)+g_{\alpha}(k) \tau_{\alpha}(E) g_{\alpha}\left(k^{\prime}\right),
$$

where the dressed $\pi N N$ form factor, $f(k ; E)$, is given by

$$
f(k ; E)=f_{0}(k)+g_{\alpha}(k) \tau_{\alpha}(E)\left\langle g_{\alpha}\left|G_{\pi N}(E)\right| f_{0}\right\rangle,
$$

and $\alpha$ in Eq. (3.7) and (3.8) refers to the $P_{11}$ channel. In Eq. (3.7), the mass renormalization factor $\Sigma(E)$ is given by

$$
\begin{aligned}
\Sigma(E) & =\left\langle f_{0}\left|G_{\pi N}(E)\right| f(E)\right\rangle \\
& =\left\langle f_{0}\left|G_{\pi N}(E)\right| f_{0}\right\rangle+\left\langle f_{0}\left|G_{\pi N}(E)\right| g\right\rangle \tau(E)\left\langle g\left|G_{\pi N}(E)\right| f_{0}\right\rangle,
\end{aligned}
$$

where we have dropped the channel label $\alpha$ with the understanding that we are considering the $P_{11}$ partial wave only.

In writing the amplitude in the $P_{11}$ channel ( Eq. (3.7) ) as the sum of a pole and a background term, we are able to define that part of the $\pi N$ amplitude which will be included in the evaluation of the three-body force. At the same time, we can determine the $\pi N N$ form factor that is required for the pion emission and absorption vertices in Fig. 1. To establish that this $\pi N N$ form factor gives the correct $\pi N N$ coupling constant as the residue of the $\pi N$ amplitude at the nucleon pole, we expand $\Sigma(E)$ about the physical nucleon mass as 44

$$
\Sigma(E)=\Sigma\left(m_{N}\right)+\left(E-m_{N}\right) \Sigma_{1}\left(m_{N}\right)+\left(E-m_{N}\right)^{2} \Sigma_{2}(E) .
$$

If we now fix the bare mass $m_{0}$ such that

$$
m_{0}+\Sigma\left(m_{N}\right)=m_{N}
$$

we can write the pole amplitude as

$$
f(k ; E) \frac{1}{E-m_{0}-\Sigma(E)} f\left(k^{\prime} ; E\right)=f^{R}(k ; E) \frac{1}{E-m_{N}} f^{R}\left(k^{\prime} ; E\right)
$$

where the renormalized $\pi N N$ form factor $f^{R}(k ; E)$ is defined as

$$
f^{R}(k ; E)=\frac{Z_{2}^{1 / 2}}{\left[1-\left(E-m_{N}\right) \sum_{2}^{R}(E)\right]^{1 / 2}} f(k ; E),
$$


and the wave function renormalization constant $Z_{2}$ is given by

$$
Z_{2}=\left[1-\Sigma_{1}\left(m_{N}\right)\right]^{-1} \equiv 1+\Sigma_{1}^{R}\left(m_{N}\right),
$$

with

$$
\Sigma_{i}^{R}(E) \equiv Z_{2} \Sigma_{i}(E) \quad \text { for } \quad i=1,2 .
$$

In this way we have defined the renormalized $\pi N N$ form factor, $f^{R}(k ; E)$, which will be used for the emission and absorption vertices in Fig. 1. More important is the fact that this form factor is constrained by the $\pi N$ phase shifts in the $P_{11}$ channel and the requirement that we have the correct $\pi N N$ coupling constant. We note at this point that the renormalized $\pi N N$ form factor $f^{R}(k ; E)$ is energy dependent and that this energy dependence is determined by unitarity through $\tau_{\alpha}(E)$, and has to be included if we are to fit the phase shifts in this channel.

To determine the $\pi N N$ coupling constant resulting from the above formulation of $\pi N$ scattering, we need to compare our results for the pole amplitude with the corresponding Feynman diagram for the Lagrangian with the pseudoscalar coupling, i.e.

$$
-i g_{0}(k) \bar{u}(\boldsymbol{\tau} \cdot \boldsymbol{\phi})\left(i \gamma_{5}\right) u \text {. }
$$

where the coupling constant $g_{0}$ is made a function of the momentum. In Eq. (3.16), $\boldsymbol{\tau}$ is the Pauli isospin matrix, $\phi$ is the pion field, and $u$ is the usual Dirac spinor. This interaction Lagrangian allows us to determine the invariant amplitude corresponding to the $s$-channel nucleon pole diagram [45], and the corresponding $S$-matrix. Making use of the relation between the $S$-matrix and the $T$-matrix [46], which is a solution of the Lippmann-Schwinger equation, we can calculate the $\pi N N$ coupling constant as a result of the relation between the $T$-matrix, $t\left(k, k^{\prime} ; E\right)$, and the invariant amplitude $\mathcal{T}\left(k, k^{\prime} ; E\right)$ as

$$
t\left(k, k^{\prime} ; E\right)=C(k ; E) \mathcal{T}\left(k, k^{\prime} ; E\right) C\left(k^{\prime} ; E\right)
$$

where

$$
C(k ; E)=\sqrt{\frac{m_{N}\left(\omega_{k}+\varepsilon_{k}\right)}{(2 \pi)^{3} \omega_{k} \varepsilon_{k}\left(E+\omega_{k}+\varepsilon_{k}\right)}},
$$

with $\varepsilon_{k}=\sqrt{k^{2}+m_{N}^{2}}$. The coupling constant is now defined as the residue of the invariant $\pi N$ amplitude at the nucleon pole with all the legs of the $\pi N N$ vertex on-mass-shell. This corresponds to taking $E=m_{N}$ and $k=k_{0}$, where

$$
k_{0}^{2}=-m_{\pi}^{2}\left(1-\frac{m_{\pi}^{2}}{4 m_{N}^{2}}\right) \text {. }
$$

This definition allows us to write the $\pi N N$ coupling constant $f_{\pi N N}$ in terms of the renormalized $\pi N N$ form factor $f^{R}(k ; E)$ as

$$
\begin{aligned}
f_{\pi N N}^{2}(k) & =\frac{m_{\pi}^{2}}{4 m_{N}^{2}} g_{0}^{2}(k) \\
& =\frac{m_{\pi}^{2}}{4 m_{N}^{2}} \frac{m_{N}\left(\varepsilon_{k}+m_{N}\right)}{6 \pi\left[C\left(k, m_{N}\right)\right]^{2}}\left[\frac{f^{R}\left(k ; m_{N}\right)}{k}\right]^{2},
\end{aligned}
$$


where

$$
\frac{1}{4 \pi} f_{\pi N N}^{2}\left(k_{0}\right)=0.079
$$

For the present investigation we make use of the $P_{11} \pi N$ parameterization of McLeod and Afnan [44, where the bare $\pi N N$ form factor $f_{0}(k)$ is taken to be

$$
f_{0}(k)=\frac{c_{0}}{\sqrt{\omega_{k}}} \frac{k}{\left(k^{2}+\alpha^{2}\right)^{n_{0}}},
$$

while for the background separable potential form factor, we take

$$
g(k)=\frac{k}{\sqrt{\omega_{k}}}\left[\frac{c_{1}}{k^{2}+\beta_{1}^{2}}+\frac{c_{2}\left(k^{2}\right)^{n_{1}}}{\left(k^{2}+\beta_{2}^{2}\right)^{3}}\right] .
$$

This choice of form factor is basically the same as that used by Thomas ( Eqs.(3.5) and

(3.6) ). The factor of $\sqrt{\omega_{k}}$ was introduced to get the covariant phase space to determine the coupling constant at the nucleon pole with all legs on-mass-shell. In Table 1 we present two parameterizations of the $P_{11}$ amplitude [44 corresponding to a monopole $\left(n_{0}=1\right)$ or a dipole $\left(n_{0}=2\right)$ bare $\pi N N$ form factor. The parameters were adjusted to give the phase shifts below the pion production threshold, the position of the nucleon pole, and the $\pi N N$ coupling constant of 0.079 . In Table II we present the scattering volume $a_{11}$ in this channel, the wave function renormalization $Z_{2}$ and the value of the coupling constant at $k=0$. We note that the values in Tables [ and $\mathbb{1}$ are the corrected values for the parameters of these potentials and their predictions. We note that the renormalized form factor $f^{R}(k ; E)$ is substantially different from the bare form factor $f_{0}(k)$ due to the wave function renormalization $Z_{2}$ and the contribution of the non-pole amplitude to the form factor dressing (see Eq.(3.8) and (3.13)). Finally, the value of the coupling constant at $k=0$, when compared with the value at $k=k_{0}$, can be used as a measure of the deviation from the Goldberger-Treiman relation.

The present definition of the $\pi N N$ form factor is different from that commonly used in $N N$ potentials, and three-nucleon force calculations. However it is consistent with the formulation of $\pi N$ scattering where the $\pi N$ amplitude is a solution to a two-body equation [47. Traditionally the $\pi N N$ form factor, introduced as a cutoff in the $N N$ amplitude, is a function of the pion momentum only. This is a result of taking both nucleons in the vertex on-mass-shell. However in a non-relativistic or time ordered theory, intermediate particles are off the energy shell. As a result, the $\pi N N$ form factor becomes a function of the energy and the relative momentum. The energy dependence of the $\pi N N$ form factor is the result of the dressing. This dressing is necessitated by the requirement that the full $\pi N$ amplitude, even in the $P_{11}$ channel, should be a solution of the Lippmann-Schwinger equation. In this way we can maintain consistency with both of the treatments for the $\pi N$ and $N N$ amplitudes as solutions of two-body equations when used in the three-nucleon system.

\section{THE THREE-BODY FORCE}

Having defined our $\pi N$ amplitude, and in particular how the nucleon pole is subtracted from the $\pi N$ amplitude to give us a $\pi N N$ form factor that is both momentum and energy 
dependent, we turn our attention to our definition of the three-body force as given in Fig. 11. At this stage it is important to note that this definition of the three-body force does not include all possible pion exchange diagrams that are not included in the nucleon-nucleon interaction. However, we expect the diagram in Fig. 1 to give the dominant contribution to the three-body force.

To evaluate the diagram in Fig. 1 we introduce Jacobi variables in the $\pi N N N$ center of mass. These are defined in Fig. 9. This choice for the momenta will allow us to take matrix elements of the three-body force between three-nucleon wave functions resulting from the solution of the Faddeev equation in momentum space for a given two-nucleon interaction. At the same time we will be able to include the $\pi N$ amplitudes defined in Sec. III with their full energy dependence with no approximations. The momenta in the initial state in Fig. 9 are

$$
\begin{aligned}
\mathbf{q}_{3} & =-\mathbf{k}_{3} \\
\mathbf{p}_{3} & =\frac{m_{N}\left(\mathbf{k}_{\pi}+\mathbf{k}_{1}^{\prime}\right)-\left(m_{N}+m_{\pi}\right) \mathbf{k}_{2}}{\left(2 m_{N}+m_{\pi}\right)} \\
\mathbf{Q}_{3} & =\frac{m_{N} \mathbf{k}_{\pi}-m_{\pi} \mathbf{k}_{1}^{\prime}}{\left(m_{N}+m_{\pi}\right)},
\end{aligned}
$$

where $\mathbf{k}_{1}^{\prime}, \mathbf{k}_{2}, \mathbf{k}_{3}$ and $\mathbf{k}_{\pi}$ are the momenta of the three nucleon and pion after the pion emission vertex. Here, $\mathrm{Q}_{3}$ is the $\pi N$ relative momentum for the pion production vertex, while $\mathrm{Q}_{3}^{\prime}$ is the relative $\pi N$ momentum in the $\pi N$ amplitude. In a similar manner we can define all the Jacobi momenta before and after the $\pi N$ scattering and before the pion absorption. In this way all momenta are defined in terms of the initial and final momenta of the three nucleons. At this stage we should point out that in a non-relativistic theory, the $\pi N N$ vertex is not Galilean invariant since mass is not conserved. As a result, the relative momenta $\mathbf{p}_{3}$ and $\mathbf{q}_{3}$ are not the same before and after the pion emission. For practical calculations we will assume that not to be the case. In other words the relative momenta $\mathbf{p}_{3}$ and $\mathbf{q}_{3}$ are those used in the three-nucleon wave function resulting from the solution of the Faddeev equations.

The pion absorption and emission vertices in Fig. 9 have the form factor $f^{R}\left(Q_{i} ; E_{i}\right), i=$ 1,3 respectively, where the energy $E_{i}$ is the energy available to the $\pi N$ system, and is given by

$$
E_{i}=E+m_{N}-\frac{q_{i}^{2}}{2 \mu_{2}}-\frac{p_{i}^{2}}{2 \mu_{1}},
$$

where $E=-E_{T}$ is the total energy of the system not including rest masses, and the reduced masses $\mu_{1}$ and $\mu_{2}$ are defined by the relations

$$
\frac{1}{\mu_{1}}=\frac{1}{m_{N}}+\frac{1}{m_{N}+m_{\pi}}, \quad \text { and } \quad \frac{1}{\mu_{2}}=\frac{1}{m_{N}}+\frac{1}{2 m_{N}+m_{\pi}} .
$$

In a similar manner, the $\pi N$ scattering in channel $\alpha$ in Fig. 9, is represented by the amplitude $t_{\alpha}\left(Q_{1}^{\prime}, Q_{3}^{\prime} ; E_{2}\right)$ which is given by

$$
t_{\alpha}\left(Q_{1}^{\prime}, Q_{3}^{\prime} ; E_{2}\right)=g_{\alpha}\left(Q_{1}^{\prime}\right) \tau_{\alpha}\left(E_{2}\right) g_{\alpha}\left(Q_{3}^{\prime}\right)
$$


where the energy available to the $\pi N$ system, $E_{2}$, is given by

$$
E_{2}=E+m_{N}-\frac{q_{3}^{2}}{2 \mu_{2}}-\frac{p_{3}^{\prime 2}}{2 \mu_{1}}=E+m_{N}-\frac{q_{1}^{2}}{2 \mu_{2}}-\frac{p_{1}^{2}}{2 \mu_{1}} .
$$

In this way we have made use of the general structure of the $\pi N$ amplitude in terms of a one-particle reducible (the $s$-channel pole amplitude) and the one-particle irreducible (the non-pole amplitude) to determine the three-body force contribution from Fig. 1. Although we have used a separable potential for the non-pole amplitude, there is no reason why we could not have made use of the non-pole contribution from a chiral Lagrangian [47, or a separable approximation to such a chiral $\pi N$ amplitude 48, other than the fact that this would have imposed an additional complexity to the evaluation of such an amplitude.

Having defined the basic ingredients required to calculate the contribution from the diagram in Fig. 1, we turn our attention to the practical problem of calculating the overall contribution of the diagram in Fig. 1. The contribution from the process whereby nucleon 1 emits a pion that scatters off nucleon 2 in the $\pi N$ channel $\alpha$, and then gets absorbed on nucleon 3 is given by the expression

$$
\begin{gathered}
W_{\beta_{1} \beta_{3}}^{\alpha}\left(p_{1}, q_{1}, p_{3}, q_{3} ; E\right)=\left\langle f^{R}\left(E_{1}\right) \mid Q_{1}\right\rangle\left\langle p_{1} q_{1}\left|G_{\pi N N N}(E)\right| p_{1}^{\prime} q_{1}\right\rangle\left\langle Q_{1}^{\prime} \mid g_{\alpha}\right\rangle \tau_{\alpha}\left(E_{2}\right) \Gamma_{\beta_{1} \beta_{3}} \\
\times\left\langle g_{\alpha} \mid Q_{3}^{\prime}\right\rangle\left\langle p_{3}^{\prime} q_{3}\left|G_{\pi N N N}(E)\right| p_{3} q_{3}\right\rangle\left\langle Q_{3} \mid f^{R}\left(E_{3}\right)\right\rangle
\end{gathered}
$$

where $\beta_{1}$ and $\beta_{3}$ represent the set of quantum numbers that label the three-body channels in the final and initial states, respectively. The coefficient $\Gamma_{\beta_{1} \beta_{3}}$ is a factor determined by the transformation between the different Jacobi momenta in the three-nucleon system. The four-body Green's function $G_{\pi N N N}(E)$ can be written in terms of the $\pi N N$ Green's function, $G_{\pi N N}$, as

$$
\begin{aligned}
G_{\pi N N N}(E) & =G_{\pi N N}\left(E-\frac{q_{i}^{2}}{2 \mu_{2}}\right) \\
& =\left(E_{i}-m_{N}-\frac{Q_{i}^{2}}{2 m_{N}}-\sqrt{Q_{i}^{2}+m_{\pi}^{2}}\right)^{-1}, \quad(i=1,3) .
\end{aligned}
$$

This allows us to employ the methods developed for pion exchange in the $N N-\pi N N$ problem and to write the partial wave projection of the process whereby nucleon 1 emits a pion that will scatter off nucleon 2 in channel $\alpha$ as

$$
\begin{aligned}
Z_{\alpha_{3}^{\prime}, \beta_{3}^{\prime}}^{j_{3}^{\prime}, t_{3}^{\prime}}\left(p_{3}^{\prime}, p_{3} ; E-\frac{q_{3}^{2}}{2 \mu_{2}}\right)= & \left\langle g_{\alpha} \mid Q_{3}^{\prime}\right\rangle\left\langle p_{3}^{\prime} q_{3}\left|G_{\pi N N N}(E)\right| p_{3} q_{3}\right\rangle\left\langle Q_{3} \mid f^{R}\left(E_{3}\right)\right\rangle \\
= & \sum_{L, a, b} A_{\alpha_{3}^{\prime}, \beta_{3}^{\prime}}^{L, a, b} p_{3}^{\ell_{\pi}-a+b} p_{3}^{\prime 1+a-b} \rho_{2}^{a+b} \\
& \quad \times \frac{1}{2} \int_{-1}^{1} d y \frac{Q_{3}^{\prime-\ell_{\pi}} g_{\alpha}\left(Q_{3}^{\prime}\right) f^{R}\left(Q_{3} ; E_{3}\right) Q_{3}^{-1}}{E_{3}-m_{N}-\frac{1}{2 m_{N}} Q_{3}^{2}-\sqrt{Q_{3}^{2}+m_{\pi}^{2}}} P_{L}(y),
\end{aligned}
$$

where $\ell_{\pi}$ is the relative $\pi N$ orbital angular momentum in the $\pi N$ amplitude. Here $\beta_{3}^{\prime}$ gives the set of quantum numbers for the coupling scheme $\left[\left(\pi N_{1}\right)_{N}, N_{2}\right]$ resulting in a total angular momentum $j_{3}^{\prime}$ and total isospin $t_{3}^{\prime}$. In a similar manner $\alpha_{3}^{\prime}=\left(\alpha, \gamma_{3}\right)$ gives the set of 
quantum numbers for the coupling scheme $\left[\left(\pi N_{2}\right)_{\alpha}, N_{1}\right]$ giving rise to the same total angular momentum $j_{3}^{\prime}$ and total isospin $t_{3}^{\prime}$. The quantum numbers of $N_{1}$ are given by $\gamma_{3}=\left(\ell_{3}^{\prime}, S_{3}^{\prime}\right)$, where $\ell_{3}^{\prime}$ is the orbital angular momentum of $N_{1}$ relative to the center of mass of $\left(\pi N_{2}\right)$, and $S_{3}^{\prime}$ ( the corresponding channel spin) is the sum of the total angular momentum $\left(j_{\pi}\right)$ of $\left(\pi N_{2}\right)$, and the spin $\left(s_{1}\right)$ of nucleon $N_{1}$, i.e., $\mathbf{S}_{3}^{\prime}=\mathbf{j}_{\pi}+\mathbf{s}_{1}$. In writing the above expression for the one pion exchange amplitude, we have made use of the fact that both $\mathbf{Q}_{3}$ and $\mathbf{Q}_{3}^{\prime}$ can be written in terms of the momenta $\mathbf{p}_{3}$ and $\mathbf{p}_{3}^{\prime}$ as

$$
\mathbf{Q}_{3}=\mathbf{p}_{3}^{\prime}+\rho_{2} \mathbf{p}_{3}, \quad \mathbf{Q}_{3}^{\prime}=\mathbf{p}_{3}+\rho_{2} \mathbf{p}_{3}^{\prime},
$$

where

$$
\rho_{2}=\frac{m_{N}}{m_{N}+m_{\pi}}
$$

and $y=\hat{\mathbf{p}}_{3} \cdot \hat{\mathbf{p}}_{3}^{\prime}$. The coefficients $A_{\alpha_{3}^{\prime}, \beta_{3}^{\prime}}^{L, a, b}$ are those used in the partial wave expansion of the Faddeev equation for a separable potential [49]. For the case when the $\pi N$ channel $\alpha$ corresponds to the $P_{33}$ partial wave, Eq. (4.10) gives the $\left(j_{3}^{\prime}, t_{3}^{\prime}\right)$ partial wave projection of the $N N-N \Delta$ transition potential. In general, since we have restricted our analysis to the case of separable non-pole $\pi N$ amplitudes, we can interpret the $\pi N$ amplitude in each partial wave to be dominated by an $N^{*}$ in which case $Z_{\alpha_{3}^{\prime}, \beta_{3}^{\prime}}^{j_{3}^{\prime}, t_{3}^{\prime}}$ can be considered as the transition potential for $N N \rightarrow N N^{*}$. The difference between the traditional $N N-N \Delta$ transition potential and the above result in Eq. (4.10) is the fact that the present transition potential is energy dependent and the parameters of the potential are determined by the $\pi N$ data rather than by the $N N$ data.

This interpretation of $Z_{\alpha_{3}^{\prime}, \beta_{3}^{\prime}}^{j_{3}^{\prime}, t_{3}^{\prime}}$ as a partial wave projected transition potential will allow us to regards the diagram in Fig. 1 to correspond to an initial state of three nucleons with nucleons 1 and 2 in the channel $\beta_{3}^{\prime}$ going to two nucleons plus an $N^{*}$, followed by the $N^{*}$ coupling to nucleon 3 to form a final state of nucleon 2 and 3 in channel $\beta_{1}^{\prime}$ with nucleon 1 as spectator. This corresponds to the exchange of an $N^{*}$ and will allow us to partial wave expand the three-body force in a similar manner to the expansion of the one pion exchange diagram treated above. The resultant partial wave expansion of the diagram in Fig. 1 is given as

$$
\begin{aligned}
W_{\beta_{1}, \beta_{3}}^{\alpha}\left(p_{1}, q_{1}, p_{3}, q_{3} ; E\right)= & \frac{1}{2} \sum_{\gamma_{1}, \gamma_{3}} \sum_{L, a, b} \int_{-1}^{1} d x(-)^{R} \frac{q_{1}^{\ell_{3}^{\prime}-a+b} q_{3}^{\ell_{1}^{\prime}+a-b}}{p_{1}^{\prime \ell_{1}^{\prime}} p_{3}^{\prime \ell_{3}^{\prime}}} \rho_{1}^{a+b} A_{\beta_{1}, \beta_{3}}^{L, a, b} P_{L}(x) \\
& \times Z_{\beta_{1}^{\prime}, \alpha_{1}^{\prime}}^{j_{1}^{\prime}, t_{1}^{\prime}}\left(p_{1}, p_{1}^{\prime} ; E-\frac{q_{1}^{2}}{2 \mu_{2}}\right) \tau_{\alpha}\left(E_{2}\right) Z_{\alpha_{3}^{\prime}, \beta_{3}^{\prime}}^{j_{3}^{\prime}, t_{3}^{\prime}}\left(p_{3}^{\prime}, p_{3} ; E-\frac{q_{3}^{2}}{2 \mu_{2}}\right),
\end{aligned}
$$

where the phase, $R=j_{\pi}+t_{\pi}+\ell_{3}^{\prime}+S_{3}^{\prime}+t_{3}^{\prime}$, results from changing the coupling scheme to maintain consistency with the definition of the Jacobi coordinates. Here, $\left(j_{\pi}, t_{\pi}\right)$ are the total angular momentum and isospin of the pion with nucleon 2, i.e., they define the $\pi N$ channel $\alpha$, while $\ell_{i}^{\prime}(i=1,3)$ are the orbital angular momenta corresponding to the momenta $p_{i}^{\prime}$, e.g. $\ell_{3}^{\prime}$ is the orbital angular momentum of $N_{1}$ relative to the center of mass of $\left(\pi N_{2}\right)$. In this case, we have written the momenta $\mathbf{p}_{3}^{\prime}$ and $\mathbf{p}_{1}^{\prime}$ in terms of the momenta $\mathbf{q}_{3}$ and $\mathbf{q}_{1}$ as 


$$
\mathbf{p}_{3}^{\prime}=\mathbf{q}_{1}+\rho_{1} \mathbf{q}_{3}, \quad \mathbf{p}_{1}^{\prime}=\mathbf{q}_{3}+\rho_{1} \mathbf{q}_{1}
$$

where

$$
\rho_{1}=\frac{m_{N}}{2 m_{N}+m_{\pi}}
$$

and $x=\hat{\mathbf{q}}_{1} \cdot \hat{\mathbf{q}}_{3}$. Here again the coefficient $A_{\beta_{1}, \beta_{3}}^{L, a, b}$ is the coefficient resulting from the angular momentum recoupling, i.e., $\left[\left(N_{1} N_{2}\right)_{\beta_{3}^{\prime}}, N_{3}\right] \rightarrow N_{1}+N_{2}^{*}+N_{3} \rightarrow\left[\left(N_{2} N_{3}\right)_{\beta_{1}^{\prime}}, N_{1}\right]$.

To compare the three-body force given in Eq. (4.13) with that resulting from the $N N-N \Delta$ coupled channel approach [25], we note that the expression in Eq. (4.13) basically consists of a transition potential $N N \rightarrow N N^{*}$ followed by the propagation of the $N^{*}$ quasiparticle and finally the transition potential $N N^{*} \rightarrow N N$. Making use of the definition of $\tau_{\alpha}\left(E_{2}\right)$, ( Eqs. (3.3) and (3.4) ), we can write this quasi-particle propagator for the case when $\alpha$ refers to the $P_{33}$ channel as

$$
\begin{aligned}
\tau_{\alpha}\left(E_{2}\right) & =\left[\left(E_{2}-E_{\Delta}\right)\left\langle g_{\alpha}\left|G_{\pi N}\left(E_{2}\right) G_{\pi N}\left(E_{\Delta}\right)\right| g_{\alpha}\right\rangle\right]^{-1} \\
& =\frac{\left[\left\langle g_{\alpha}\left|G_{\pi N}\left(E_{2}\right) G_{\pi N}\left(E_{\Delta}\right)\right| g_{\alpha}\right\rangle\right]^{-1}}{\left[E+m_{N}-E_{\Delta}-\frac{q_{3}^{2}}{2 \mu_{2}}-\frac{p^{\prime 2}}{2 \mu_{1}}\right]} .
\end{aligned}
$$

In writing this equation we have made use of the fact that $\tau_{\alpha}^{-1}\left(E_{\Delta}\right)=0$, where $E_{\Delta}=$ $m_{\Delta}-\frac{i}{2} \Gamma=1230-50 i \mathrm{MeV}$ is the position of the $\Delta(1230)$ resonance, and $\alpha$ refers to the $P_{33}$ partial wave. This illustrates the fact that our quasi-particle propagator is the free $N N \Delta$ propagator for which the $\Delta$ has a width, and this width has the correct energy dependence as dictated by unitarity and the experimental phase shifts in this channel. Thus if we include the contribution to the three-body force from the $P_{33} \pi N$ channel only, we have effectively included, to lowest order, the contribution from the three-body force resulting from the $N N-N \Delta$ coupling. However, our result differs from the standard definition of this contribution to the three-body force 225] in that we have employed the $\pi N$ data rather than the $N N$ data to fix the parameters of this force, and our $\Delta$ is a proper resonance in the $\pi N$ system and not a real particle as it is often considered. The other difference between this approach and that used in the $N N-N \Delta$ coupled channel approach is in the choice of the pion propagator in the "transition potential". In the $N \Delta$ coupled channel approach, the pion propagator is taken to be $\left(k_{\pi}^{2}+m_{\pi}^{2}\right)^{-1}$, which corresponds to the Feynman propagator with the nucleons on-mass-shell. This propagator has no energy dependence. On the other hand we have chosen the standard non-relativistic four particle Green's function with a relativistic expression for the pion kinetic energy. This is consistent with four-particle unitarity and is equivalent to a time ordered propagator, and to that extent we have only one time order for our pion exchange. Finally, we should note that the $N N-N \Delta$ coupled channel approach treats the contribution of the $\Delta$ to all orders and as a result includes the dispersive contribution which to a large extent cancels the contribution of the three-body force.

The recent three-nucleon force results, reported by Peña et al. [27], treat the $\Delta$ as a $\pi N$ resonance. To that extent, the $\pi N$ amplitude in the $P_{33}$ channel is similar to that presented here, in that the mass and width of the $\Delta$ have energy dependence as dictated 
by $\pi N$ scattering data. Peña et al. have the additional advantage that they not only have included the $\Delta$ contribution to the three-body force to all orders, but have also included the dispersive contribution. However, by restricting their Hilbert space to $N$ and $\Delta$ and turning off all interaction in the pionic part of the Hilbert space, they have not included the contribution of the $\pi N$ amplitude in other than the $P_{33}$ partial wave. They find that the contribution of the non-resonant $\pi N$ amplitude is very small as a result of the fact that the pionic component of the three-nucleon wave function is small.

We now try to relate our three-body force with that used in the TM approach. In the TM approach, the final $\pi N$ amplitude is written in terms of the pion momenta, where as we use the $\pi N$ relative momenta $\mathbf{Q}_{1}^{\prime}$ and $\mathbf{Q}_{3}^{\prime}$ [50]. To achieve their result, we recall that the partial wave expansion of the $\pi N$ amplitude is given by

$$
\left\langle\mathbf{Q}|t(E)| \mathbf{Q}^{\prime}\right\rangle=\sum_{\alpha} P_{\alpha}\left(\hat{\mathbf{Q}}, \hat{\mathbf{Q}}^{\prime}\right) t_{\alpha}\left(Q, Q^{\prime} ; E\right)
$$

where $\alpha=(\ell, j, T)$ are the quantum numbers corresponding to the orbital and total angular momentum and isospin of the $\pi N$ system, i.e. $\alpha$ labels the different partial waves. The partial wave projection operator $P_{\alpha}\left(\hat{\mathbf{Q}}, \hat{\mathbf{Q}}^{\prime}\right)$ is defined as

$$
P_{\alpha}\left(\hat{\mathbf{Q}}, \hat{\mathbf{Q}}^{\prime}\right)=P_{T} \sum_{m}\left\langle\hat{\mathbf{Q}} \mid \mathcal{Y}_{\ell j m}\right\rangle\left\langle\mathcal{Y}_{\ell j m} \mid \hat{\mathbf{Q}}^{\prime}\right\rangle
$$

with $P_{T}$ the projection operator for a given isospin channel, and $\left\langle\hat{\mathbf{Q}} \mid \mathcal{Y}_{\ell j m}\right\rangle$ the eigenstates of the orbital and total angular momentum of the $\pi N$ system. If we now write the angular momentum projection operator in terms of $\mathbf{Q} \cdot \mathbf{Q}^{\prime}$ and $\mathbf{Q} \times \mathbf{Q}^{\prime} \cdot \boldsymbol{\sigma}$ and the isospin projection operator in terms of the Pauli isospin operator, we can write our $\pi N$ amplitude, assuming the pion scatters of nucleon 1, in the form presented by the Tucson-Melbourne formulation as

$$
t^{T M}\left(\mathbf{Q}, \mathbf{Q}^{\prime}\right)=\left(\boldsymbol{\tau}_{2} \cdot \boldsymbol{\tau}_{3}\right)\left[a+b \mathbf{Q} \cdot \mathbf{Q}^{\prime}+c\left(Q^{2}+Q^{\prime 2}\right)\right]+d\left(\boldsymbol{\tau}_{1} \cdot\left(\boldsymbol{\tau}_{3} \times \boldsymbol{\tau}_{2}\right)\right)\left(\boldsymbol{\sigma}_{1} \cdot\left(\mathbf{Q} \times \mathbf{Q}^{\prime}\right)\right)
$$

For the factors $a, b, c$ and $d$ to be constant, as required by the Tucson-Melbourne definition of the three-body force, we have to make the following approximations: (i) Since the TM potential is derived from the off-mass-shell $\pi N$ amplitude $T\left(\nu, t, q, q^{\prime}\right)$, where $t$ is the Mandelstam variable and $q\left(q^{\prime}\right)$ is the four momentum of the initial (final) pion, by expanding the amplitude about $\nu=0$, we need to determine the corresponding approximation for our off-energy-shell $\pi N$ amplitude. For the off-mass-shell amplitude the nucleon pole $\left(s=m_{N}^{2}\right)$ traces a curve in the $\nu-t$ plane that crosses the $\nu$-axes close to $\nu=0$ [51]. Since we find it difficult to directly relate the variables that in the off-mass-shell amplitude with the corresponding variables in off-energy-shell amplitude, we have chosen the position of the nucleon pole, i.e. $E=m_{N}$ or $s=m_{N}^{2}$, to be the closest approximation to $\nu=0$. In this way we approximate the energy in our amplitude to be the nucleon mass, i.e., $\tau\left(E_{2}\right) \rightarrow \tau\left(m_{N}\right)$. (ii) The separable potential form factors $g_{\alpha}(Q)$ have to be expanded in a power series in the momentum $Q$, keeping those terms such that the final amplitude does not have any powers of the momentum higher than the momentum squared. With these approximations, the Thomas separable potential, in conjunction with the potential $P J$ in the $P_{11}$ channel, can 
be written in the above form with the constants $a, b, c$ and $d$ given in Table III. Included in the table are also the corresponding parameters from the Tucson-Melbourne potential [15].

In next section, we will present our numerical result and show: (i) How the contribution to the three-body force from each $\pi N$ partial wave depends on the energy in the $\pi N$ amplitude. (ii) How the three-nucleon force is sensitive to the choice of the $\pi N N$ form factor which is determined as the residue of the $P_{11}$ pole term. Though we have not included all the diagrams that would contribute to the three-body force, we have included the most important contribution, which will allow us to examine these effects.

\section{NUMERICAL RESULTS}

Having defined our three-nucleon force in terms of the $\pi N$ potential whose parameters have been adjusted to fit the $\pi N$ data, and in particular, the phase shifts up to the threshold for pion production, we turn our attention in this section to the calculation of the contribution of this force to the binding energy of the three-nucleon system. As a first calculation with a three-body potential that includes the energy dependence of the $\pi N$ amplitude, we have chosen to use the first order perturbation theory to calculate this three-body force contribution. Therefore, we can write our three-body force as

$$
\begin{aligned}
W & =\sum_{\substack{i, j=1 \\
i \neq j}}^{3} W(j, i) \\
& =\sum_{\substack{i, j=1 \\
i \neq j}}^{3} \sum_{\alpha} W_{j, i}^{\alpha}\left(\mathbf{p}_{j}, \mathbf{q}_{j} ; \mathbf{p}_{i}^{\prime}, \mathbf{q}_{i}^{\prime}\right),
\end{aligned}
$$

where $j$ in the sum refers to the nucleon that emits the pion, and $i$ refers to the nucleon that absorbs the pion. Here, $\alpha$ refers to the $\pi N$ partial wave used to calculate the three-body force. Since the three-nucleon wave function used is a solution of the Faddeev equations, we can write this wave function as the sum of three components, i.e.

$$
\begin{aligned}
|\Psi\rangle & =\left|\psi_{1}\right\rangle+\left|\psi_{2}\right\rangle+\left|\psi_{3}\right\rangle \\
& =[1+(123)+(132)]\left|\psi_{1}\right\rangle,
\end{aligned}
$$

where we have written the total wave function in terms of the elements of the permutation operators. Making use of the properties of the permutation group, we can write the total contribution to the binding energy from this three-body force as

$$
\Delta E^{(3)}=6\langle\Psi|W(1,3)| \Psi\rangle .
$$

The three-body potential $W(1,3)$ can now be partial wave expanded in terms of the partial wave potential given in Eq. (4.13) as

$$
\left\langle\mathbf{p}_{1} \mathbf{q}_{1}|W(1,3)| \mathbf{p}_{3} \mathbf{q}_{3}\right\rangle=\sum_{\alpha} \sum_{\beta_{1} \beta_{2}}\left\langle\hat{\mathbf{p}}_{1} \hat{\mathbf{q}}_{1} \mid \beta_{1}\right\rangle W_{\beta_{1} \beta_{3}}^{\alpha}\left(p_{1}, q_{1}, p_{3}, q_{3} ; E\right)\left\langle\beta_{3} \mid \hat{\mathbf{p}}_{3} \hat{\mathbf{q}}_{3}\right\rangle,
$$

where $\beta_{i}$ defines the three-body partial wave quantum number in which nucleon $i$ is the spectator. In Eq. (5.4), $\alpha$ refers to the $\pi N$ partial wave that contributes to the three-body 
potential. In a similar manner we have to expand the three-nucleon wave function in terms of the angular momentum and isospin bases $\left\langle\hat{\mathbf{p}}_{1} \hat{\mathbf{q}}_{1} \mid \beta_{1}\right\rangle$. This is given by

$$
\begin{aligned}
\left\langle\mathbf{p}_{1} \mathbf{q}_{1} \mid \Psi\right\rangle & =\sum_{\beta_{1}}\left\langle\hat{\mathbf{p}}_{1} \hat{\mathbf{q}}_{1} \mid \beta_{1}\right\rangle\left\langle\beta_{1} ; p_{1} q_{1} \mid \Psi\right\rangle \\
& =\sum_{\beta_{1}=1}^{N_{2}}\left\langle\hat{\mathbf{p}}_{1} \hat{\mathbf{q}}_{1} \mid \beta_{1}\right\rangle\left[\left\langle p_{1} q_{1} \mid \psi_{\beta_{1}}\right\rangle+\sum_{j=2,3} \sum_{\beta_{j}=1}^{N_{1}}\left\langle\beta_{1} \mid \beta_{j}\right\rangle\left\langle p_{j} q_{j} \mid \psi_{\beta_{j}}\right\rangle\right] .
\end{aligned}
$$

In Eq. (5.5) the sum over $N_{1}$ extends to the number of three-body channels included in the solution of the Faddeev equations which in turn is determined by the number of two-body $N N$ channels included. On the other hand, the sum over $N_{2}$ is an infinity sum which we have truncated for practical calculations. For $N_{2}>N_{1}$ the first term $\left\langle p_{1} q_{1} \mid \psi_{\beta_{1}}\right\rangle$ contributed only to the first $N_{1}$ terms in the $N_{2}$ sum. This restriction is the result of truncating the number of $N N$ partial waves included in the solution of the Faddeev equations. We will test the convergence of our final results to both the sum over $N_{1}$ and $N_{2}$. Making use of the partial wave expansion for the three-body potential and the three-nucleon wave function, we can write the total contribution of the three-body force to the binding energy of the triton as

$$
\begin{aligned}
\Delta E^{(3)}=6 \sum_{\alpha} & \sum_{\beta_{1} \beta_{3}} \int_{0}^{\infty} d p_{1} p_{1}^{2} \int_{0}^{\infty} d p_{3} p_{3}^{2} \int_{0}^{\infty} d q_{1} q_{1}^{2} \int_{0}^{\infty} d q_{3} q_{3}^{2} \\
& \times\left\langle\Psi \mid p_{1} q_{1} ; \beta_{1}\right\rangle W_{\beta_{1} \beta_{3}}^{\alpha}\left(p_{1}, q_{1}, p_{3}, q_{3} ; E\right)\left\langle\beta_{3} ; q_{3} p_{3} \mid \Psi\right\rangle,
\end{aligned}
$$

where $E$ is the energy of the three-nucleon system as determined by the solution of the Faddeev equations for a given two-nucleon interaction, and the partial wave three-body potential, $W_{\beta_{1} \beta_{3}}^{\alpha}$, as given in Eq. (4.13).

In the present investigation we have chosen to use the Paris nucleon-nucleon potential [52]. The Paris potential is energy independent while our three-nucleon force has been derived to be energy-dependent. To that extent, our two- and three-body potentials are not consistent in that they are not derived from the same Lagrangian. However, since we are examining the energy dependence of the three-body force for each $\pi N$ partial wave, we hope that the present perturbative calculation may allow us to gain some insight into this problem. To simplify the construction of the three-nucleon wave function needed to evaluate the integrals in Eq. (5.6), we have chosen the separable representation of the Paris potential [32]. This representation has been tested for the three-nucleon observables with considerable success [53]. In Table IV], we give the rank of the separable expansion we have chosen. This choice was dictated by the requirement that we should reproduce the binding energy of the triton and the different percentages of $S-, S^{\prime}-$, and $D$-state probability for the triton. However, before we compare our results with the coordinate space calculation for the Paris potential, we present in Table $\nabla$ the convergence of these quantities as we increase the number of three-body channels, $N_{1}$, in the solution of the Faddeev equations. From this table we can see that as far as the three-nucleon wave function is concerned, the 18 channel Faddeev equations give good convergence for all quantities. These 18 channels in the Faddeev equations correspond to the truncation of the $N N$ interaction to include all two-body partial waves with total angular momentum less than or equal to two, including the coupled ${ }^{3} P_{2}-{ }^{3} F_{2} N N$ channels. To justify the use of the separable expansion to the Paris potential, 
PEST [32], we compare, in Table V1, our results for the 18 channel calculation with the corresponding results based on the coordinate space solution of the Faddeev equations for the Paris potential. Taking the difference between the two coordinate space calculations as a measure of the numerical uncertainty in the solution of the Faddeev equations, we have good agreement with previous results for the Paris potential. This suggests that the three-nucleon wave function resulting from the PEST approximation is comparable to that resulting from a solution of the Faddeev equations in coordinate space for the exact Paris potential.

Having established the fact that the three-nucleon wave function generated by the separable expansion to the Paris potential is of comparable quality to that resulting from the solution of the Faddeev equations in coordinate space, we turn our attention to the convergence of the contribution of the three-body force to the number of three-body channels in the solution of the Faddeev equations, $N_{1}$, and the number of three-body channels included in the partial wave expansion of the wave function, $N_{2}$. For this study we make use of the $P_{11}$ potential PJ of McLeod and Afnan [44]. In Table VI] we present the contribution to the binding energy from the more important $\pi N$ partial waves for 5, 10, and 18 channel Faddeev calculations. In all cases we have taken 18 partial waves for the expansion of the wave function. All energies in Table VII are in $\mathrm{keV}$. We note at this stage that although the contribution to the binding energy is small, the 18 channel Faddeev calculation has converged, while the 5 channel calculation gives an incorrect result. With the 18 channel Faddeev calculation, we tested the convergence of our result to the number of terms in the partial wave expansion of the three-body wave function, $N_{2}$. From the results in Table VIII we may conclude that $N_{2}=18$ is sufficient to give us a $1 \mathrm{keV}$ accuracy for the contribution from a given $\pi N$ partial wave. If the need arises we might have to resort to more terms in the partial wave expansion of the wave function.

In Table $[\mathrm{X}]$ we present the contribution of the three-body force to the binding energy of the triton from the different $\pi N$ partial waves for two different $P_{11}$ potentials. Here we have taken $N_{1}=N_{2}=18$, with all energies given in keV. The most surprising result of our calculations is the overall small contribution of the three-body force. From the results in Tables VII and VIII, it is clear that the inclusion of more three-body partial waves in the wave function expansion and the solution of the Faddeev equation will not change the results substantially. Before we address the origin of this small contribution from our threenucleon force it is interesting to note: (i) The comparable contribution from the $S$ - and $P$-waves $\pi N$ partial wave, and in particular the large contribution of the $S_{31}$ compared to the $S_{11}$. This suggests that we need to include both $S$ - and $P$-wave $\pi N$ amplitudes into the calculation. Furthermore, models based on the dominance of the $\Delta(1230)$ resonance might not be valid since they neglect the contribution from the $S_{31}$ and $P_{11}$ partial waves. In fact, in the present formulation, the contribution of the $P_{33}$ partial wave amplitude is for $\pi N$ energies below the nucleon pole, some $300 \mathrm{MeV}$ below the $\Delta$ resonance. (ii) There is a cancellation between the $S$ - and $P$-wave $\pi N$ contributions requiring a consistent treatment of both sets of partial wave amplitudes. (iii) The $P$ - wave contribution comes equally from the non-pole part of the $P_{11}$, and the $P_{33}$ partial wave amplitudes. This is despite the fact that the overall $P_{11}$ phase shifts are small when compared with the $P_{33}$ phase shifts. However, if we recall that it is the non-pole part of the amplitude that contributes to the three-body force, and this non-pole part, on its own, has phase shifts that are comparable to those in the $P_{33}$ channel [54,55], then the results reported in Table [X] are not surprising. 
Finally, if we compare the results for the two potentials, we find that the potential $M 1$ gives a larger contribution to the binding energy than the potential $P J$. To understand this difference, we compare the dressed form factors for these two potentials in Fig. 10. Here we observe that the potential $M 1$ has a harder form factor than the potential $P J$, i.e., the dressed form factor for potential $M 1$ is greater than the corresponding form factor for the potential $P J$ for large $k$. This is consistent with results of the fact that the three-body force contribution to the binding energy increases as the form factor gets harder. We will come back to this point later in our discussion when we consider the role of the $\pi N N$ form factor in the contribution of the three-nucleon force to the binding energy of the triton.

We now turn to the question of why the contribution of this three-body force is small. From Table III, we may expect the maximum difference between

our prediction and the TM result for the three-body force contribution to be at most an order of magnitude, but not three orders of magnitude. Since the unique feature of the present calculation is the inclusion of the energy dependence in both the $\pi N N$ form factor and the $\pi N$ amplitude, we will commence by turning this energy dependence off. We will also concentrate on those $\pi N$ partial waves that give a substantial contribution to the threebody force. We will restrict our results to $N_{1}=N_{2}=18$. As a first approximation, denoted (i) in Table $囚$, we fix the energy in the $\pi N N$ form factor to be the position of the nucleon pole, i.e.

$$
f^{R}(k ; E) \rightarrow f^{R}\left(k ; m_{N}\right) .
$$

Although this approximation changes our final result by increasing the contribution of the three-body force to the binding energy, the magnitude of the increase is not substantial because the cancellation between the repulsive $S_{31}$ and the attractive $P_{11}$ and $P_{33}$ contributions is still present. In particular, we note that both the attractive and repulsive contributions have increased in magnitude. We next take the energy dependence in the $\pi N$ amplitude to be the position of the nucleon pole (approximation (ii)), i.e.

$$
\tau_{\alpha}(E) \rightarrow \tau_{\alpha}\left(m_{N}\right)
$$

In this case, we have increased the total contribution of the three-body force to the binding energy by an order of magnitude as compared to the exact result. This substantial increase in binding is mainly due to the fact that the $S_{31}$ contribution is reduced in magnitude while the $P_{11}$ and $P_{33}$ contributions have increased, thus reducing the cancellation between the attraction and repulsion when compared with the exact calculation. To understand why the contribution of the $S_{31}$ partial wave is suppressed as the energy in $\tau_{\alpha}$ is increased (i.e. brought closer to the $\pi N$ threshold, see Fig. 3), we recall from Eqs. (3.3) and (3.4) that $\lambda=+1$ for repulsive potentials such as the $S_{31}$, while $\lambda=-1$ for attractive potentials such as the $P_{33}$. On the other hand, $\left\langle g_{\alpha}\left|G_{\pi N}(E)\right| g_{\alpha}\right\rangle$ is negative for $E<\left(m_{\pi}+m_{N}\right)$ and increases in value as we approach the $\pi N$ threshold from below. Thus for attractive potentials there is a cancellation in the denominator of $\tau_{\alpha}(E)$ giving rise to an increase in the value of $\tau_{\alpha}(E)$ as $E$ approaches the threshold. On the other hand, for repulsive potentials, e.g. the $S_{31}$ channel, the value of $\tau_{\alpha}(E)$ decreases as we approach the elastic threshold from below, resulting in a suppression of the repulsive contribution. To demonstrate the validity of this argument, we have proceeded to change the energy in the $\pi N$ amplitude, to be the threshold for $\pi N$ scattering, i.e., 


$$
\tau_{\alpha}(E) \rightarrow \tau_{\alpha}\left(m_{N}+m_{\pi}\right) .
$$

The result of this approximation is labeled (iii) in Table $X$. Here, we observe that the contribution of the $S_{31}$ is further reduced, while the $P_{11}$ and $P_{33}$ contributions are increased in magnitude, giving even more attraction. We now fix the energy of both the $\pi N N$ form factor and the $\pi N$ scattering amplitude to the nucleon pole, i.e. $E=m_{N}$, (i) \& (ii) in Table \. This gives even more binding than fixing the energy in either the $\pi N$ scattering amplitude or the $\pi N N$ form factor. Finally, we can increase binding further by fixing the energy of the $\pi N N$ form factor at the nucleon pole, while the energy in the $\pi N$ amplitude is taken to

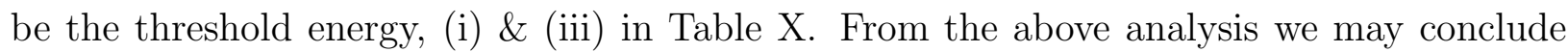
that it is the energy dependence in both the $\pi N N$ form factor and the $\pi N$ amplitude that has substantially reduced the contribution of the three-nucleon force to the binding energy of the triton, and this reduction is a result of the cancellation between the repulsive $S_{31}$ contribution and attractive $P_{11}$ and $P_{33}$ contributions. However, the approximation of fixing the energy in the $\pi N$ amplitude and $\pi N N$ form factor does not give us a sufficiently large contribution which is comparable with the result for the TM potential.

Finally, to fully understand the origin of this small contribution to the binding energy from the three-nucleon force, we turn our attention to the form factors used in the separable potential and the form factor in the $\pi N N$ vertex. It is common practice to take the $\pi N N$ form factor in $N N$ scattering to be either of a dipole or monopole form. Therefore, as a first step in examining the sensitivity of our final three-body force contribution to the binding energy, we replace the $\pi N N$ form factor $f^{R}(k ; E)$ by a monopole, i.e.

$$
\left.f^{R}(k ; E) \rightarrow \frac{f^{R}\left(k ; m_{N}\right)}{k}\right|_{k=0} k F_{0}(k),
$$

where the monopole form factor $F_{0}(k)$ is given by

$$
F_{0}(k)=\frac{\Lambda^{2}}{\Lambda^{2}+k^{2}}
$$

with the cutoff mass $\Lambda$, varied. In Table XI we compare exact results for the $P_{11}$ potential $P J$ [44] with the result for the approximations in Eqs. (5.7) and (5.8), referred to as (i) \& (ii), and the approximations in Eqs. (5.8) and (5.10) with $\Lambda=400$ and $800 \mathrm{MeV}$. The results in lines 2, 3 and 4 of Table $\mathrm{X}]$ have the energy in the $\pi N$ amplitude fixed to be the energy at the nucleon pole, i.e. $E_{\pi N}=m_{N}$. Here we observe that the final total contribution of the three-body force increases by an order of magnitude when the energy in both the $\pi N N$ form factor and $\pi N$ amplitude is fixed at the nucleon pole. There is a further order of magnitude increase when the $\pi N N$ form factor is replaced by a monopole form factor with a cutoff mass of 400 and then $800 \mathrm{MeV}$. In fact, half of this increase is achieved when the cutoff mass is increased from 400 to $800 \mathrm{MeV}$. This establishes the sensitivity of our result to the choice of $\pi N N$ form factor. In this case it is interesting to observe that the $S_{31}$ and $P_{11}$ contributions to the three-nucleon force almost completely cancel, leaving the $P_{33}$ contribution to be approximately the total contribution. Thus in this approximation, the contribution to the three-nucleon force is predominantly due to the channel in which the $\Delta(1230)$ dominates the scattering amplitude. This result should be compared with the exact results, line 1 of Table $\mathrm{X} 1$, where the $S_{31}$ contribution cancels the sum of the $P_{11}$ and $P_{33}$ contributions. 
In the spirit of the TM approach, the $\pi N$ amplitude is expanded to lowest order in $\frac{q}{m_{N}}$ and a monopole $\pi N N$ form factor is introduced. This approximation may be implemented by the following replacement:

$$
\begin{aligned}
f^{R}(k ; E) & \left.\rightarrow \frac{f^{R}\left(k ; m_{N}\right)}{k}\right|_{k=0} k F_{0}(k) \\
\tau_{\alpha}(E) & \rightarrow \tau_{\alpha}\left(m_{N}\right) \\
g_{\alpha}(k) & \left.\rightarrow \frac{g_{\alpha}(k)}{k^{\ell}}\right|_{k=0} k^{\ell} F_{0}(k),
\end{aligned}
$$

where $\ell$ is the angular momentum in channel $\alpha$ and the monopole form factor $F_{0}(k)$ is defined in Eq. (5.11). However in our formulation, this replacement will destroy the fit to the experimental $\pi N$ phase shifts. In Table XI1, we compare our exact result for the $P_{11}$ potential PJ [44], with the results of the approximation in Eq. (5.12) with $\Lambda=400$ and $800 \mathrm{MeV}$. The approximation in Eq. (5.12) gives rise to an increase in the contribution of the $P$-waves substantially, while the $S$-wave contribution remains relatively unchanged, and therefore negligible. In fact we can now adjust the cutoff mass $\Lambda$ to get the difference between the experimental binding energy and the calculated three-nucleon result for any of the two-nucleon interactions.

To understand this large change in the magnitude of the total contribution of the threebody force when we introduce the form factor $F_{0}(k)$, we compare in Fig. 10 the monopole form factor with $\Lambda=400$ and $800 \mathrm{MeV}$ and the dressed $\pi N N$ form factor for the potentials $P J$ and $M 1$. Here we observe that the form factor $F_{0}(k)$ with $\Lambda=800 \mathrm{MeV}$ is almost a factor of 3 larger than the dressed $\pi N N$ form factor at $k \approx 3 \mathrm{fm}^{-1}$. Furthermore, this form factor comes raised to the power of two in the case when only the $\pi N N$ form factor is replaced by a monopole form factor, or a power of four when the approximations in Eq. (5.12) are implemented, i.e. we have a power of two from the $\pi N N$ vertices and another power of two from the form factor of separable $\pi N$ amplitudes. Thus the difference between the result of including $f^{R}\left(k, m_{N}\right)$ and $F_{0}(k)$ should be roughly one order of magnitude in Tables XI, and two orders of magnitude in Table XII. In the latter case we assume the separable potential form factors have similar ranges to the dressed $\pi N N$ form factor. This explains the drastic change in the contribution of the three-body force to the binding energy when we introduced the monopole form factor $F_{0}(k)$ into our calculation.

Finally, to get the closest approximation to the TM three-nucleon force, we have modified the propagator for the exchanged pion by replacing our propagator by the corresponding Feynman propagator, i.e.,

$$
\frac{1}{Q_{0}-\omega_{Q}} \rightarrow \sqrt{2 \omega_{Q}} \frac{1}{Q^{2}-m_{\pi}^{2}} \sqrt{2 \omega_{Q}},
$$

where $\omega_{Q}=\sqrt{Q^{2}+m_{\pi}^{2}}$. To understand the difference between these two propagators, we recall that the Feynman propagator has both a positive energy and negative energy component, since

$$
\frac{1}{Q^{2}-m_{\pi}^{2}}=\frac{1}{2 \omega_{Q}}\left[\frac{1}{Q_{0}-\omega_{Q}}-\frac{1}{Q_{0}+\omega_{Q}}\right]
$$


and our choice of propagator, i.e. $\left(Q_{0}-\omega_{Q}\right)^{-1}$, corresponds to taking the positive energy component of the Feynman propagator. However in any energy independent approximation, the Feynman propagator reduces to $-1 / \omega_{Q}^{2}$. The substitution in Eq. (5.13) gives us the last line of Table XII, and results in the reduction of the contribution of the three-body force so that a monopole form factor with a cutoff mass of $800 \mathrm{MeV}$ will give a three-body force contribution of about $0.6 \mathrm{MeV}$, which is consistent with the results reported in the literature for the TM three-nucleon force.

Thus, to get a substantial contribution from the three-body force we have had to make two approximations. (i) We have dropped the energy dependence of the $\pi N$ amplitude and the $\pi N N$ form factor. (ii) We have modified the off-shell behavior of the $\pi N$ amplitude by introducing the same monopole form factor in all partial waves, at the sacrifice of the fit to the experimental data, in order to get a substantial increase to the three-body force contribution. Although the first approximation is not justified, the second could be accepted on the ground that we have chosen the wrong off-shell behavior. In particular, we should consider changes the cutoff mass in the bare $\pi N N$ form factor $f_{0}(k)$, and make use of the Goldberger-Treiman [13] relation to constrain the dressed $\pi N N$ form factor $f^{R}(k ; E)$. This point is presently under further investigation.

\section{CONCLUSION}

The main motivation for this investigation was to establish the relative contribution of the different partial waves of the $\pi N$ amplitude in determining the $\pi-\pi$ three-nucleon force and its contribution to the binding energy of the triton. To achieve this, we made use of a separable potential formulation of the $\pi N$ scattering, taking into consideration that such a parameterization of the off-shell $\pi N$ amplitude has been used in $\pi d$ scattering and in the derivation of the pion-nucleus optical potential with considerable success. In particular, we maintained the energy dependence of the $\pi N$ amplitude since that could effect the overall contribution to the three-body force. More importantly, the cancellation between the contribution of the different partial waves could be sensitive to the inclusion of this energy dependence. Since the energy domain, important to the determination of the three-nucleon force, is in the unphysical region and below the position of the nucleon pole in the energy plane, we chose to fit the scattering data closest to this region. In particular we chose to fit the scattering lengths, the phase shifts up to the pion production threshold, and the position and the residue of the $\pi N$ amplitude at the nucleon pole. This parameterization allowed us to determine the $\pi N N$ form factor and that part of the $\pi N$ amplitude that gives rise to the three-body force.

From the numerical results of our calculation we can conclude that: (i) The energy dependence of both the $\pi N N$ form factor and $\pi N$ amplitude gives a suppression of the contribution of Fig. 1 to the three-nucleon force. Given the fact that this diagram has always been considered to give the main contribution to this force, we can conclude that the three-nucleon force for this $\pi N$ parameterization is small, and will not change substantially

if we include this three-body force in an exact calculation rather than in the perturbative approach used in the present investigation. (ii) The inclusion of the energy dependence gives rise to a substantial cancellation between the contribution from the repulsive $S_{31}$ partial wave and the attractive $P_{11}$ and $P_{33}$ partial waves. (iii) The contribution from the $P_{33}$ partial 
wave is not as dominant as we would expect. In fact the attraction comes equally from the $P_{11}$ and $P_{33}$, while the repulsion comes from the $S_{31}$. This raises a question about the validity of including the three-body force in terms of the $N N-N \Delta$ coupled channel approach while neglecting the $S$-wave and the $P_{11} \pi N$ contributions. (iv) The choice of the $\pi N N$ form factor, to be determined by the residue of the $\pi N$ amplitude at the nucleon pole, is the other main reason for the reduction in the overall magnitude of the three-nucleon force contribution to the binding energy. The question of the possibility of choosing the cutoff mass in the bare form factor $f_{0}(k)$ to be consistent with the $\pi N$ data, while maintaining a substantial three-body force, will need further investigation. Furthermore, the GoldbergerTreiman relation [13,14] should be used to constrain the momentum dependence of the dressed $\pi N N$ form factor $f^{R}(k ; E)$.

To further substantiate the above conclusions, we may need to examine a number of questions: (i) How sensitive are our results to the choice of the $\pi N$ interaction? In particular, would we get a small contribution from the three-nucleon force, and specifically the diagram in Fig. 1, if we commenced with a chiral Lagrangian such as that used by Pearce and Jennings [47]? (ii) Would our final results be substantially different if this three-body force were to be included to all orders? Examination of the perturbation series for the Paris potential with the TM three-body force [56] suggests that higher order contributions are not negligible, but the magnitude of the overall contribution does not change by more than a factor of two. This further suggests that an exact calculation will not effect our final conclusion. (iii) How important are the dispersive effects? The latest results based on the $N N-N \Delta$ coupled channel [25] approach suggests that there is a substantial cancellation between the three-body force contribution and the dispersive effects in the $P_{33}$ channel. What happens to this cancellation when other $\pi N$ partial waves are included? If this cancellation is present for all $\pi N$ partial waves then it would lead to further reduction in the three-body force. 


\section{ACKNOWLEDGMENT}

The authors would like to thank the Australian Research Council and Flinders University Board of Research for their financial support during the course of this work. The authors would also like to thank J. Haidenbauer for supplying them with the parameters of the PEST potential, and D.R. Lehman for the comparison of triton results for the PEST potential. Finally we would like to thank B.F. Gibson for supplying us with the results of the coordinate space calculation for the triton with the Paris potential. 


\section{REFERENCES}

[1] B. F. Gibson, Nucl. Phys. A543, 1c (1992).

[2] T. Sasakawa and S. Ishikawa, Few-Body Systems 1, 3 (1986).

[3] S. Ishikawa and T. Sasakawa, ibid. 1, 143 (1986).

[4] J. L. Friar, B. F. Gibson and G. L. Payne, Phys. Rev. C 37, 2869 (1988).

[5] J. L. Friar, G. L. Payne, V. G. J. Stoks and J. J. de Swart, Phys. Lett. B 311, 4 (1993).

[6] W. N. Polyzou and W. Glöckle, Few-Body Systems, 9, 97 (1990).

[7] J. Carlson, V. R. Pandharipande and R. B. Wiringa, Nucl. Phys. A401, 59 (1983).

[8] R. Schiavilla, J. Carlson, V. R. Pandharipande and R. B. Wiringa, Nucl. Phys. A449, 219 (1986).

[9] V. R. Pandharipande, "The Three-Body Force in the Three-Nucleon System" Ed. B. L. Berman and B. F. Gibson, Springer-Verlag p 59 (1986).

[10] S. A. Coon , M. D. Scadron, P. C. McNamee, B. R. Barrett, D. W. E. Blatt, and B. H. J. McKellar, Nucl. Phys, A317, 242 (1979).

[11] S. A. Coon and W. Glöckle, Phys. Rev. C 23, 1790 (1981).

[12] G. E. Brown, A. M. Green and W. J. Gerace, Nucl. Phys. A115, 435 (1968).

[13] M. L. Goldberger and S. B. Treiman, Phys. Rev. 110, 1178 (1958).

[14] S. A. Coon and M. D. Scadron, Phys. Rev. C 42, 2256 (1990).

[15] S. A. Coon and M. T. Peña, Phys. Rev. C 48, 2559 (1993).

[16] H. T. Coelho, T. K. Das and M. R. Robilotta, Phys. Rev. C 28, 1812 (1983).

[17] M. R. Robilotta and M. P. Isido Filho, Nucl. Phys. A414, 394 (1984).

[18] M. R. Robilotta and H. T. Coelho, Nucl. Phys. A460, 645 (1986).

[19] S. A. Coon and J. L. Friar, Phys. Rev. C 34, 1060 (1986).

[20] S. Weinberg, Phys. Lett. B251, 288 (1990); Nucl. Phys. B363, 3 (1991); Phys. Lett. B295, 114 (1992).

[21] Ch. Hajduk, P. U. Sauer and W. Strueve, Nucl. Phys. A405, 581 (1983).

[22] Ch. Hajduk, P. U. Sauer and S. N. Yang, Nucl. Phys. A405, 605 (1983).

[23] P. U. Sauer, Prog. Part. Nucl. Physics 16, 35 (1986).

[24] M. T. Peña, H. Henning and P. U. Sauer, Phys. Rev. C 42, 855 (1990).

[25] A. Picklesimer, R. A. Rice, and R. Brandenburg, Phys. Rev. C 44, 1359 (1991); Phys. Rev. C 45, 547 (1992); Phys. Rev. Lett. 68, 1484 (1992); Phys. Rev. C 45, 2045 (1992); Phys. Rev. C 46, 1178 (1992).

[26] A. Stadler and P. U. Sauer, Phys. Rev. C 46, 64 (1992).

[27] M. T. Peña, P. U. Sauer, A. Stadler and G. Kortemeyer, Phys. Rev. C 48, 2208 (1993).

[28] Y. Avishai and T. Mizutani, Nucl. Phys. A393, 429 (1983).

[29] G. Cattapan, L. Canton and J. P. Svenne, Nuov. Cimento 106 A, 1229 (1993).

[30] Since the three nucleon wave function is determined within the framework of the nonrelativistic Faddeev equations, where all particles are on-mass-shell and all amplitudes are off-energy-shell, we will consider $\pi N N$ dynamics and therefore the $\pi N$ amplitude is in an off-energy-shell formulation.

[31] P. U. Sauer, M. Sawicki and S. Furui, Prog. Theo. Phys. 74, 1290 (1985).

[32] J. Haidenbauer and W. Plesas, Phys. Rev. C 30, 1822 (1984).

[33] T. Mizutani and D. S. Koltun, Ann. Phys. (N.Y.) 109, 1 (1977)

[34] H. Feshbach, Ann. Phys. (N.Y.) 19, 287 (1962).

[35] A. O. Alt, P. Grassberger and W. Sandhas, Nucl. Phys. B2, 167 (1967) 
[36] J. A. Elsey and I. R. Afnan, Phys. Rev. D 40, 2353 (1989)

[37] B. Blankleider and A. N. Kvinikhidze, Few-Body Systems Suppl. 7, 294 (1994)

[38] A. W. Thomas, Adv, Nucl. Phys. 13, 1 (1984).

[39] A. W. Thomas, J. Phys. G7, L283 (1981).

[40] A. W. Thomas, Nucl. Phys. A258, 417 (1976).

[41] B. Blankleider and I. R. Afnan, Phys. Rev. C 24, 1572 (1981).

[42] I. R. Afnan and R. J. McLeod, Phys. Rev. C 31, 1821 (1985).

[43] I. R. Afnan and A. T. Stelbovics, Phys. Rev. C 23, 1384 (1981).

[44] R. J. McLeod and I. R. Afnan, Phys. Rev. C 32, 222 (1985); C 32, 1786E (1985).

[45] J. D. Bjorken and S. D. Drell, Relativistic Quantum Mechanics McGraw-Hill (N.Y.) 1964).

[46] I. R. Afnan and A. W. Thomas, in Modern Three-Hadron Physics Ed. A. W. Thomas, Springer-Verlag (1976).

[47] B. C. Pearce and B. K. Jennings, Nucl. Phys. A528, 655 (1991).

[48] B. C. Pearce and I. R. Afnan Phys. Rev. C 40, 220 (1989).

[49] I. R. Afnan and B. F. Gibson, Phys. Rev. C 41, 2787 (1990).

[50] The $\pi N$ amplitudes used in the present investigation are a function of the $\pi N$ relative momenta $\mathbf{Q}_{1}^{\prime}$ and $\mathbf{Q}_{3}^{\prime}$, while the TM amplitude is a function of the pion momenta $\mathbf{k}_{\pi}$ and $\mathbf{k}_{\pi}^{\prime}$. In the static limit, the relative $\pi N$ momenta reduces to the pion momenta. In comparing the structure of our amplitude to that of the TM amplitude, we have ignored this difference.

[51] S.A.Coon, Few Body Systems, Supp. 1, 41 (1986).

[52] M. Lacombe, B. Loiseau, J. M. Richard, R. Vinh Mau, J. Côté, P. Pirès, and R. de Tourreil, Phys. Rev. C 21, 861 (1980).

[53] W. C. Parke, Y. Koike, D. R. Lehman, and L. C. Maximon, Few-Body Systems, 11, 89 (1991).

[54] T. Mizutani, C. Fayard, G. H. Lamot and S. Nahabetian, Phys. Rev. C 242633 (1981).

[55] S. Morika and I. R. Afnan, Phys. Rev. C 26, 1148 (1982).

[56] A. Bömelburg, Phys. Rev. C 34, 14 (1986). 


\section{FIGURES}

FIG. 1. The contribution to the three-nucleon force.

FIG. 2. The $N N$ complex energy plane with the unitarity cut and the domain of integration when used in the Faddeev equation for the three-nucleon system.

FIG. 3. The $\pi N$ complex energy plane with the unitarity cut, the $N$ and $\Delta$ pole position, and the region of integration when calculating the three-body $\pi-\pi$ force.

FIG. 4. The dressing of nucleons with the restriction on the Hilbert space to include nucleons and up to one pion only.

FIG. 5. The full dressing of nucleons on both sides of the $\pi N N$ vertex.

FIG. 6. The lowest order terms resulting from the coupling of the $N N N$ to the $\pi N N N$ channels as given in Eq.(2.17). (a) One pion exchange term, $\mathcal{V}_{O P E}$. (b) Dispersion term, $\mathcal{V}_{\text {disp }}$. And (c) three body force, $\mathcal{V}_{3 B}$.

FIG. 7. The nucleon pole contribution to the $\pi N$ potential.

FIG. 8. The contribution of the crossed diagram and the contact diagram to the $\pi N$ potential.

FIG. 9. An illustration of the Jacobi momenta defined for the three-body force.

FIG. 10. Comparison of the dressed $\pi N N$ form factors for the potentials $P J$, and $M 1$ with a monopole form factor having cutoff mass of $\Lambda=400,800 \mathrm{MeV}$. 


\section{TABLES}

TABLE I. Parameters of the McLeod-Afnan $P_{11}$ potentials.

\begin{tabular}{l|ccrrrrrrr}
\hline \hline Pot. & $n_{0}$ & $n_{1}$ & \multicolumn{1}{c}{$c_{0}$} & $c_{1}$ & $c_{2}$ & \multicolumn{1}{c}{$\begin{array}{c}\alpha \\
\mathrm{fm}^{-1}\end{array}$} & $\begin{array}{c}\beta_{1} \\
\mathrm{fm}^{-1}\end{array}$ & \multicolumn{1}{c}{$\beta_{2}$} & \multicolumn{1}{c}{$\begin{array}{l}m_{0} \\
\mathrm{fm}^{-1}\end{array}$} \\
\hline$P J$ & 2 & 1 & 43.5646 & 0.2907 & 412.97 & 3.8206 & 1.2688 & 5.181 & 5.1574 \\
$M 1$ & 1 & 2 & 1.0726 & 0.3433 & 2.54 & 2.7703 & 1.4422 & 2.1982 & 5.4314 \\
\hline \hline
\end{tabular}

TABLE II. The scattering volume $a_{11}$, wave function renormalization $Z_{2}$, and the $\pi N N$ coupling constant at $k=0$ for the $P_{11}$ potentials. The $f_{\pi N N}(0)$ is to be compared with a value of 0.2726 predicted by the Goldberger-Treiman relation of $3 \%$ change in $f_{\pi N N}(k)$ between $k=k_{0}$ and $k=0$.

\begin{tabular}{l|ccc}
\hline \hline Pot. & $a_{11}$ & $Z_{2}$ & $f_{\pi N N}(0)$ \\
& $\left(m_{\pi}^{-3}\right)$ & & 0.2280 \\
\hline$P J$ & -0.0706 & 0.8059 & 0.2209 \\
$M 1$ & -0.0721 & 0.7273 & 0.2209 \\
\hline \hline
\end{tabular}

TABLE III. The parameters of the Tucson-Melbourne type $\pi N$ amplitude as reported in Ref. [15], and those extracted from the potential of Thomas [40] and the $P_{11}$ potential PJ [44].

\begin{tabular}{l|cccc}
\hline \hline Pot. & $a$ & $b$ & $c$ & $d$ \\
\hline Peña \& Coon & 1.03 & -2.62 & 0.91 & -0.75 \\
Thomas \& PJ & 0.46 & -4.66 & 0.67 & -2.47 \\
\hline \hline
\end{tabular}

TABLE IV. The rank of the separable expansion to the Paris potential for the two-body $N N$ partial waves included in the calculation of the wave function.

\begin{tabular}{l|ccccc}
\hline \hline & ${ }^{1} S_{0}$ & ${ }^{3} S_{1^{-}}{ }^{3} D_{1}$ & ${ }^{3} P_{0},{ }^{1} P_{1},{ }^{3} P_{1}$ & ${ }^{3} P_{2^{-}}{ }^{3} F_{2}$ & ${ }^{1} D_{2},{ }^{3} D_{2}$ \\
\hline Rank & 3 & 4 & 2 & 3 & 2 \\
\hline \hline
\end{tabular}


TABLE V. The convergence of the binding energy of the triton, the $S-S^{\prime}-$ and $D$-state probabilities for 5, 10 and 18 channel Faddeev calculations. In calculating the wave function we have taken $N_{2}=146$.

\begin{tabular}{c|rrrr}
\hline \hline$N_{1}$ & B.E. $(\mathrm{MeV})$ & $P(S) \%$ & $P\left(S^{\prime}\right) \%$ & $P(D) \%$ \\
\hline 5 & 7.2659 & 89.882 & 1.652 & 8.401 \\
10 & 7.0966 & 90.274 & 1.479 & 8.182 \\
18 & 7.3175 & 90.111 & 1.430 & 8.393 \\
\hline \hline
\end{tabular}

TABLE VI. Comparison of our 18 channel results for the separable expansion (PEST), with the results of coordinate space calculations of Los Alamos (LA) [4] and Sendai (S) [2].

\begin{tabular}{l|cccr}
\hline \hline Model & B.E. $(\mathrm{MeV})$ & $P(S) \%$ & $P\left(S^{\prime}\right) \%$ & $P(D) \%$ \\
\hline PEST & 7.318 & 90.111 & 1.430 & 8.393 \\
LA & 7.388 & 90.130 & 1.395 & 8.409 \\
S & 7.56 & 90.17 & 1.32 & 8.45 \\
\hline \hline
\end{tabular}

TABLE VII. Convergence of the contribution of the three-nucleon force to the binding energy of the triton from different $\pi N$ partial waves and for different numbers of the three-body channels in the Faddeev equations. Here we have taken $N_{2}=18$. All energies are in keV.

\begin{tabular}{l|rrrr}
\hline \hline$N_{1}$ & $S_{11}$ & $S_{31}$ & $P_{11}$ & $P_{33}$ \\
\hline 5 & -5.5 & 31.8 & -7.7 & -15.2 \\
10 & -4.8 & 26.5 & -7.9 & -15.0 \\
18 & -4.8 & 26.4 & -8.8 & -16.0 \\
\hline \hline
\end{tabular}

TABLE VIII. Convergence of the contribution of the three-nucleon force to the binding energy from different $\pi N$ partial waves as a function of $N_{2}$, the number of three-body channels in the partial wave expansion of the wave function. The Faddeev equations are solved with 18 channels. All energies are in $\mathrm{keV}$.

\begin{tabular}{c|rrrr}
\hline \hline$N_{2}$ & $S_{11}$ & $S_{31}$ & $P_{11}$ & $P_{33}$ \\
\hline 10 & -5.2 & 37.1 & -7.2 & -8.5 \\
18 & -4.8 & 26.4 & -8.8 & -16.0 \\
26 & -5.3 & 26.2 & -9.5 & -16.1 \\
34 & -5.3 & 25.7 & -9.5 & -16.9 \\
\hline \hline
\end{tabular}


TABLE IX. The contribution to the three-body force from different $\pi N$ partial wave amplitudes. The results are for two different choices of the $P_{11}$ potential. All energies are in keV.

\begin{tabular}{c|ccccccc}
\hline \hline Pot. & $S_{11}$ & $S_{31}$ & $P_{11}$ & $P_{31}$ & $P_{13}$ & $P_{33}$ & Total \\
\hline$P J$ & -4.8 & 26.4 & -8.8 & -3.6 & 4.5 & -16.0 & -2.3 \\
$M 1$ & -5.0 & 28.9 & -15.3 & -2.1 & 6.2 & -22.1 & -9.4 \\
\hline \hline
\end{tabular}

TABLE X. The effect of removing the energy dependence in the $\pi N N$ form factor (i), and the $\pi N$ amplitudes, (ii) and (iii). The results in this table are for the $P_{11}$ potential $P J$, and all energies are in $\mathrm{keV}$.

\begin{tabular}{c|rrrrrrr}
\hline \hline Approx. & $S_{11}$ & $S_{31}$ & $P_{11}$ & $P_{31}$ & $P_{13}$ & $P_{33}$ & Total \\
\hline exact & -4.8 & 26.4 & -8.8 & -3.6 & 4.5 & -16.0 & -2.3 \\
(i) & -5.9 & 31.7 & -13.0 & -3.0 & 5.7 & -22.3 & -6.8 \\
(ii) & -5.0 & 23.5 & -13.7 & -3.6 & 3.9 & -28.2 & -23.1 \\
(iii) & -5.7 & 19.5 & -19.6 & -3.5 & 3.6 & -49.8 & -55.5 \\
(i) \& (ii) & -6.1 & 28.1 & -20.1 & -3.1 & 5.0 & -39.8 & -36.0 \\
(i) \& (iii) & -7.0 & 23.4 & -28.7 & -3.0 & 4.7 & -70.3 & -80.9 \\
\hline \hline
\end{tabular}

TABLE XI. The effect of changing the $\pi N N$ form factor on the contribution of the three-nucleon force to the binding energy of the triton. The energy in the $\pi N$ amplitude is fixed at $m_{N}$. The comparison is between monopole form factor with a cutoff mass $\Lambda=400$ or $800 \mathrm{MeV}$ and $f^{R}\left(k, m_{N}\right)$. Also included are the exact results which have the full energy dependence of both the $\pi N N$ form factor and $\pi N$ amplitude. The total includes the contributions from all $S$ - and $P$-wave $\pi N$ amplitudes. All energies are in keV.

\begin{tabular}{c|ccccc}
\hline \hline$\Lambda$ & $S_{11}$ & $S_{31}$ & $P_{11}$ & $P_{33}$ & Total \\
\hline exact & -4.8 & 26.4 & -8.8 & -16.0 & -2.3 \\
(i) \& (ii) & -6.1 & 28.1 & -20.1 & -39.8 & -36.0 \\
400 & -12.5 & 57.6 & -40.7 & -81.2 & -68.7 \\
800 & -29.3 & 147.5 & -204.1 & -364.0 & -395.1 \\
\hline \hline
\end{tabular}


TABLE XII. The effect of changing the form factors on the contribution of the three-nucleon force to the binding energy of the triton. Here, both the $\pi N N$ form factors $f^{R}(k ; E)$ and the separable potential form factors $g_{\alpha}(k)$ are replaced by a monopole with a cutoff mass $\Lambda=400$ or $800 \mathrm{MeV}$. The last line in this table corresponds to taking the 'Feynman' propagator for the pion. The total includes the contributions from all $S$ - and $P$-wave $\pi N$ amplitudes. All energies are in $\mathrm{keV}$.

\begin{tabular}{c|rrrrr}
\hline \hline$\Lambda$ & $S_{11}$ & $S_{31}$ & $P_{11}$ & $P_{33}$ & Total \\
\hline exact & -4.8 & 26.4 & -8.8 & -16.0 & -2.3 \\
400 & -15.0 & 18.8 & -58.8 & -211.5 & -231.1 \\
800 & -82.7 & 113.2 & -1146.7 & -3172.5 & -3897.2 \\
$800^{*}$ & -36.0 & 47.4 & -193.4 & -542.5 & -611.6 \\
\hline \hline
\end{tabular}


This figure "fig1-1.png" is available in "png" format from: http://arxiv.org/ps/nucl-th/9412004v1 
This figure "fig2-1.png" is available in "png" format from: http://arxiv.org/ps/nucl-th/9412004v1 
This figure "fig3-1.png" is available in "png" format from: http://arxiv.org/ps/nucl-th/9412004v1 
This figure "fig1-2.png" is available in "png" format from: http://arxiv.org/ps/nucl-th/9412004v1 
This figure "fig2-2.png" is available in "png" format from: http://arxiv.org/ps/nucl-th/9412004v1 
This figure "fig3-2.png" is available in "png" format from: http://arxiv.org/ps/nucl-th/9412004v1 
This figure "fig1-3.png" is available in "png" format from: http://arxiv.org/ps/nucl-th/9412004v1 
This figure "fig2-3.png" is available in "png" format from: http://arxiv.org/ps/nucl-th/9412004v1 
This figure "fig3-3.png" is available in "png" format from: http://arxiv.org/ps/nucl-th/9412004v1 
This figure "fig2-4.png" is available in "png" format from: http://arxiv.org/ps/nucl-th/9412004v1 